\title{
Identification of attractive blend for spotted wing drosophila, Drosophila suzukii, from apple juice
}

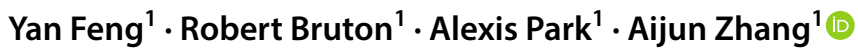

Received: 24 October 2017 / Revised: 29 May 2018 / Accepted: 16 June 2018 / Published online: 22 June 2018

(C) The Author(s) 2018

\begin{abstract}
Drosophila suzukii, commonly known as the spotted wing drosophila (SWD), is an exotic fruit fly from Southeast Asia that was introduced to the temperate regions of North America and Europe in 2008. It attacks a wide variety of fruits and has become a devastating pest of soft-skinned fruit crops. Due to the rapid spread of SWD across the newly invaded continents, fresh fruit markets have a zero-tolerance policy regarding $D$. suzukii infestation. Specific and efficient $D$. suzukii detection tools are urgently needed so that farmers can deliver timely management interventions to meet market demands. Since SWD is known to be attracted to damaged and rotting fruits, headspace volatiles from fresh and fermented apple juices were collected and analyzed by gas chromatography-mass spectrometry. Special attention was given to the compounds produced and/ or enriched during the fermentation process. After performing a series of laboataory and field tests, we identified a quinary blend, which is more efficient and selective for $D$. suzukii than the currently standard apple cider vinegar and commercially available SWD lure under field conditions. Identification of SWD attractant will help growers accurately detect $D$. suzukii adult infestations in orchards, thereby allowing for timely pest management interventions while reducing conventional insecticidal usage to protect our crops, environment, and ecosystem.
\end{abstract}

Keywords Fruit fly $\cdot$ Attractant $\cdot$ Fermentation $\cdot$ Acetoin $\cdot$ Ethyl octanoate $\cdot$ Insect pest management

\section{Key message}

- Drosophila suzukii attacks a wide variety of fruits and has become a devastating pest of soft-skinned fruit crops.

- Early detection of this fruit fly on farms is essential for quick management measures that could lead to reductions in the rate and amount of insecticide applications.

- A quinary blend was identified from the headspace volatiles of apple juice.

Communicated by A. Biondi.

Electronic supplementary material The online version of this article (https://doi.org/10.1007/s10340-018-1006-9) contains supplementary material, which is available to authorized users.

Aijun Zhang

aijun.zhang@ars.usda.gov

1 Invasive Insect Biocontrol and Behavior Laboratory, Agricultural Research Service, United States Department of Agriculture, Bldg. 007, Rm. 312, BARC-W, Beltsville, MD 20705, USA
- This blend is more efficient and selective for attraction of D. suzukii than the currently standard apple cider vinegar (ACV) under field conditions.

\section{Introduction}

The spotted wing drosophila (SWD), Drosophila suzukii Matsumura (Diptera: Drosophilidae), is an invasive fruitinfesting fly native to Southeast Asia (Calabria et al. 2010). Since it was accidently introduced to central California in August 2008, D. suzukii has rapidly spread across the USA (Hauser 2011). It has also been detected in Canada, Mexico, and Europe (Asplen et al. 2015; Lee et al. 2011). Drosophila suzukii attacks a wide variety of fruits and has become a devastating pest of soft-skinned fruit crops such as raspberries, blueberries, cherries, and strawberries (Beers et al. 2011; Hauser 2011). Unlike most drosophilid flies that feed and oviposit on overripe, damaged, or decomposing fruits, D. suzukii can feed and oviposit on sound ripening fruits (Calabria et al. 2010; Hamby et al. 2016; Mitsui et al. 2006). The females possess a serrated ovipositor, which allows 
them to cut through the epicarp of their hosts. Fruit infestation by $D$. suzukii larvae has resulted in significant financial losses to farmers (Cini et al. 2012; Walsh et al. 2011).

Due to its economic impact on fruit crops (Goodhue et al. 2011), farmers usually resort to calendar-based applications of insecticides to manage $D$. suzukii (Beers et al. 2011; Lee et al. 2011). Detection of this fly on farms is essential for quick management measures, which will reduce the rate and amount of insecticide applications required to avoid economic loss. Since Drosophila spp. are strongly attracted to overripe, fallen, rotting fruit (Mallis 1969), traps baited with fermentation products such as apple cider vinegar (ACV), wine, or yeast as baits are employed for SWD populations monitoring on farms (Beers et al. 2011; Hamby and Becher 2016; Landolt et al. 2012b; Lee et al. 2012). In particular, ACV is commonly used because it is easily available and relatively cheap (Beers et al. 2011; Lee et al. 2011); however, ACV is not adequately selective and effective for SWD detection (Tonina et al. 2018). In studies testing different trap designs using ACV as bait, it was found that only 26-31\% of the total numbers of captured Drosophila were D. suzukii (Lee et al. 2012) and high numbers of two beneficial parasitoids were found in the traps (Wang et al. 2016). In addition, ACV-baited traps are ineffective at detecting flies before populations reach an economic injury level leaving farmers without sufficient time to apply protective treatments (Burrack et al. 2015; Lee et al. 2012). Therefore, many researches focused on identification of the key attractive compounds for D. suzukii from wine, yeast, and SWD preferred fruits have been carried out. Although 11-component blend based on raspberry volatile (Abraham et al. 2015) and eight-component (Cha et al. 2012) and four-component (Cha et al. 2014, 2015) blends based on wine and yeast volatiles and some commercial synthetic lures have been developed and tested as SWD attractants, they are unsatisfactory for the demands of SWD adult infestation detection and population monitoring because of their poor selectivity and efficiency (Basoalto et al. 2013; Burrack et al. 2015; Cha et al. 2013; Iglesias et al. 2014; Kirkpatrick et al. 2017; Kleiber et al. 2014; Mazzetto et al. 2015; Tonina et al. 2018). Current monitoring systems suffer from inconsistent efficacy, and they exhibit variability in trap captures depending on crop type, crop phenology, and D. suzukii phenology and are difficult to relate trap captures to infestation (Hamby and Becher 2016). More effective and selective attractants are needed for detecting, monitoring, and managing this invasive species.

This work focuses on developing more effective and selective attractants for $D$. suzukii based on volatiles from fruit aromas. Since the yeast microbes volatiles from ripening fruits could induce strong attraction in Drosophila larvae and flies (Becher et al. 2010, 2012; Hamby and Becher 2016), headspace volatiles collected from fresh and fermented apple juices were compared. Special attention was given to the compounds produced and/or enriched during the fermentation process. This newly identified quinary chemical blend is expected to lure SWD flies more efficiently and selectively into traps in the early season and attract them to insecticide strips or biocontrol dispensers. It will be vital for SWD adult infestation detection and population monitoring in support of SWD management programs. The new blend will provide opportunity to enable future development of mass trapping and attract-and-kill technologies for control of this exotic pest.

\section{Materials and methods}

\section{Apple juice headspace volatile collections and raspberry solvent extract preparation}

The headspace of apple juice was collected using fresh and fermented Old Orchard ${ }^{\circledR}$ brand apple juice (100\% apple juice from concentrate with vitamin C; no sugar, artificial flavors, colors, or preservatives added; Shoppers Food and Pharmacy, College Park, MD) in August 2013. Fresh apple juice $(250 \mathrm{~mL}$, obtained from store and used right away) was introduced into a 1-L four-necked glass container (Zhang et al. 1994, 1999). Air was drawn into the container through 6-14-mesh activated charcoal (Fisher Scientific, Pittsburgh, $\mathrm{PA})$ and out of the container through two traps $(15 \mathrm{~cm} \times 1.5$ cm o.d.) containing Super Q (200 mg each; Alltech Associates, Inc., Deerfield, IL) by vacuum $(\sim 1 \mathrm{~L} / \mathrm{min})$. For fresh apple juice volatile collection, aeration was conducted for $3 \mathrm{~h}$ at room temperature in daytime. Adsorbents were eluted with methylene chloride $(4 \times 0.5 \mathrm{~mL})$; the elutes $(2 \mathrm{~mL} /$ each sample) were concentrated to $\sim 500 \mu 1$ under a nitrogen stream and stored at $-30{ }^{\circ} \mathrm{C}$ for future gas chromatography (GC)-mass spectrometry (MS) analysis. For fermented apple juice volatile collection, the same container was set on the laboratory benchtop for 20 days while the apple juice fermentation occurred inside (ACV changed into unclear and turbid solution). Aeration was then conducted for $3 \mathrm{~h}$, and adsorbent elution was carried out in the same manner as fresh apple juice volatile. A polydimethylsiloxane-coated solid-phase microextraction (SPME) fiber (PDMS. $100 \mu \mathrm{m}$, Supelco Inc., Bellefonte, Pennsylvania) was used for apple juice SPME sampling (Zhang et al. 1999). Raspberry extract was prepared at Rutgers University from fresh raspberry fruit by homogenized blending and centrifuging (Abraham et al. 2015). The resulting extracts containing water-soluble organic substances were stored in a freezer $\left(-10^{\circ} \mathrm{C}\right)$ for laboratory bioassay (Abraham et al. 2015). 


\section{GC and GC-MS spectrometry}

The GC and electronic impact (EI) GC-MS systems used were as described (Zhang et al. 2004). A Hewlett Packard (HP) $6890 \mathrm{GC}$ was coupled to a flame ionization detector (FID) using DB-WAXETR or DB-5 capillary column $(60 \mathrm{~m} \times 0.25 \mathrm{~mm}$ i.d., $0.25-\mu \mathrm{m}$ film thickness, J\&W Scientific Inc., Folsom, CA). Oven temperature was started at $50{ }^{\circ} \mathrm{C}$ for $2 \mathrm{~min}$, then programmed to rise to $230^{\circ} \mathrm{C}$ at $15^{\circ} \mathrm{C} /$ $\mathrm{min}$, and held for $20 \mathrm{~min}$ in the splitless mode with hydrogen as carrier ( $2 \mathrm{~mL} / \mathrm{min})$. For GC-MS analysis, a HP $6890 \mathrm{GC}$ was coupled to a HP 5973 Mass Selective Detector (MSD) using the same columns as GC-FID, but with helium as carrier $(1.4 \mathrm{~mL} / \mathrm{min})$. A $70 \mathrm{eV}$ electron beam was employed for sample ionization. The chemical identification of the headspace volatiles was based on comparison of their mass spectra with the NIST and Wiley mass spectral libraries, and identities were confirmed by mass spectra and GC retention times of authentic standards on both polar and nonpolar GC capillary columns (Zhang et al. 1999). The ratios of identified components were determined by using GC-FID.

\section{Insect rearing}

Laboratory-reared D. suzukii flies were obtained from Rutgers University in 2014 with permission (permit: P526P14-00,267) from the Animal and Plant Health Inspection Service (APHIS). The original colony was established in July 2012 from $D$. suzukii-infested high-bush blueberry Bluecrop cv. (Vaccininum corymbosum L.) fruits in Burlington County, New Jersey (Abraham et al. 2015). Insects were reared on cornmeal diet (Dalton et al. 2011) in polystyrene tubes (height $95 \mathrm{~mm}$, diameter $28.5 \mathrm{~mm}$, Fisher Scientific, PA, USA) with ventilated plugs (height $25 \mathrm{~mm}$, diameter $28.5 \mathrm{~mm}$, Fisher Scientific, PA, USA). The colony was maintained in an incubator under $\sim 25^{\circ} \mathrm{C}, 60 \% \mathrm{RH}$, and a $16: 8$-h (L/D) photoperiod. Maintenance of the D. suzukii colony was achieved by transferring emerging adults into new diet tubes on a weekly basis. All $D$. suzukii used in bioassays (2-8 days post-eclosion) were counted and sexed based on the presence of a dark spot on the wing tips of males and the presence of a serrated ovipositor in females (Walsh et al. 2011) for overall colony sex ratio determination. The sex ratio of laboratory-reared $D$. suzukii was determined to be close to 1:1.

\section{Chemicals}

All chemicals used in this work were purchased from SigmaAldrich (St. Louis, MO, USA), including isobutanol (IB), $99 \geq \%$, CAS 78-83-1; 2-methyl-1-butanol (2 MB), $99 \geq \%$, CAS 137-32-6; 3-methyl-1-butanol (3 MB), 98.5 $\geq \%$, CAS 123-51-3; ethyl hexanoate (EH), 99 $\geq \%$, CAS 123-66-0; acetoin (AT), 99\%, CAS 512-86-0; ethyl octanoate (EO), $99 \geq \%$, CAS 106-32-1; acetic acid (AA), $99.7 \geq \%$, CAS 64-19-7; benzaldehyde (BA), 98 $\geq \%$, CAS 100-52-7; ethyl decanoate (ED), 99 $\geq \%$, CAS 110-38-3; methyl benzoate (MB), 99\%, CAS 93-58-3; phenethyl alcohol (PE), $99 \geq \%$, CAS 60-12-8; as well as ethanol (EtOH), 200 proof HPLC grade, ethyl acetate (EA), methylene chloride, and pentane, HPLC grade. They were used without further purification. Pentane was used as a solvent for dilution when lower-than-stock concentrations of any of the aforementioned chemicals were required.

\section{Field tests}

Commercially available Victor ${ }^{\circledR}$ Yellowjacket \& Flying Insect Traps (Great Lakes IPM Inc., Vestaburg, MI) filled with $\sim 300 \mathrm{~mL}$ of tap water containing a surfactant (Seventh Generation ${ }^{\mathrm{TM}}$ Natural Dish Liquid-Free \& Clear, Shoppers Food and Pharmacy, College Park, MD, $4 \mathrm{~mL} /$ gallon) as a drowning solution were used in all field tests at the Beltsville Agricultural Research Center-West, Beltsville, MD (10300 Baltimore Ave., Beltsville, MD) and at the blueberry field at Butler's Orchard (22222 Davis Mill $\mathrm{Rd}$, Germantown, MD). No pesticide was sprayed during the trapping experiments. Unless otherwise indicated, pure chemical (individual or blend, $1 \mathrm{~mL}$ ) was loaded onto a cotton ball held within a polypropylene flex micro-centrifuge tube (1.5-mL Eppendorf micro-centrifuge tube, VWR International, Radnor, PA), and the lid of the tube was then closed. Tubes filled only with a cotton ball were used as blank controls. The micro-centrifuge tube lure was opened (S1 Fig.), and the lid was snapped into one of four holes on the cap of the Victor ${ }^{\circledR}$ trap, leaving the three remaining holes to serve as entrances for attracted insects (S2 and S3 Fig.). At the Beltsville Agricultural Research Center, traps were hung from the branches of trees (S4 Fig.) on the edge of a small woodlot in blocks consisting of 3-5 treatments and one blank control with the traps spaced approximately $5 \mathrm{~m}$ apart within a group. Three to 15 replications were tested for each treatment (for ethanol effect: $N=10$, Fig. 4a, and acetic acid effect: $N=15$, Fig. 4b, during October 2015; for ethyl octanoate effect: $N=3$, Fig. 4 c, during November 2015; for ethyl acetate effect: $N=3$, Fig. 4 d, during November 2016; for comparison with Scentry commercial lure: $N=6$, Fig. 5a, b, during November and December 2016; for phenethyl alcohol effect: $N=6$, Fig. 5c, during December 2016). The treatments were randomly arranged at different positions weekly within the block, and blocks were separated by $10-20 \mathrm{~m}$. At the blueberry field in Butler's Orchard, traps were hung from the branches of blueberry bushes, deployed in blocks consisting of different treatments with the traps spaced approximately $5 \mathrm{~m}$ apart in a group. Nine to 12 replications were tested for each treatment (for a quinary blend 
test: $N=9$, Fig. $5 \mathrm{~d}$, during December 2016; for comparison with ChemTica commercial lure: $N=12$, Table 3, during July 2017). The traps were randomly arranged at different positions within the block, and blocks were separated by $10-20 \mathrm{~m}$.

The preliminary field tests were conducted at the Beltsville Agricultural Research Center during October 17-24,
2014, using six different lures: (1) apple juice blend [treatment 1 , seven components based on apple juice fermentation products (Fig. 1)], (2) raspberry blend (EAG active 11-component blend) (Abraham et al. 2015), (3) single component [2-methyl butanol (2 MB)], (4) [3-methyl butanol (3 MB)], (5) binary components blend [( $2 \mathrm{MB}+3 \mathrm{MB}$ in 1:1 ratio)], and (6) blank control (Table 1, $N=3$ ). Field-collected $D$.

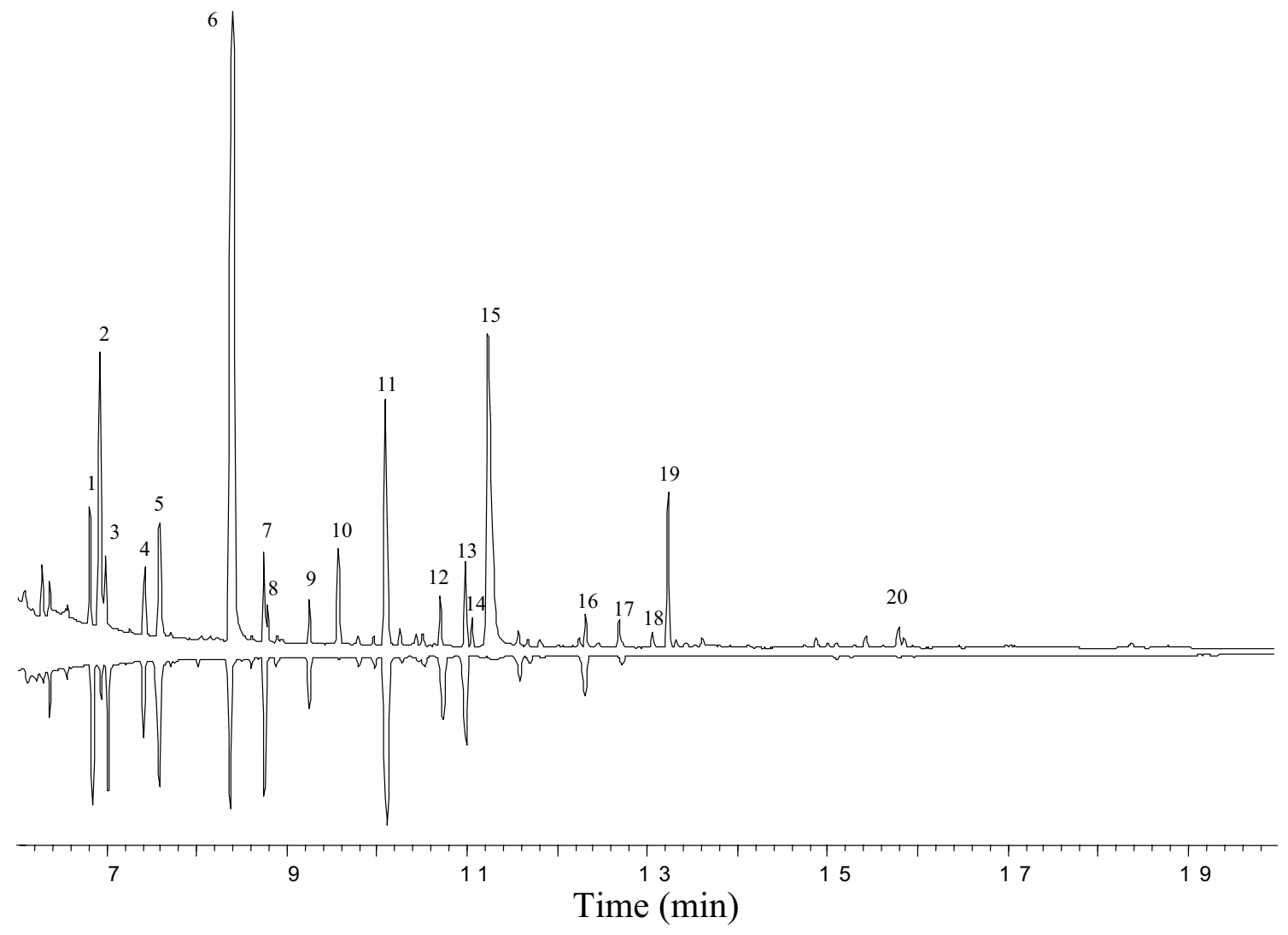

Fig. 1 GC-MS traces (total ion) of apple juice volatile extracts of fermented (top) versus fresh (bottom) on DB-Waxetr capillary column. 1. Butyl acetate, 2. isobutanol, 3. hexanal, 4. 2-methylbutyl acetate, 5. butanol, 6. 2- and 3-methyl butanol, 7. trans-2-hexenal, 8. ethyl hexanoate, 9. hexyl acetate, 10. 3-hydroxy-2-butanone (acetoin), 11. hexanol, 12. trans-2-hexenol, 13. unknown. 14. ethyl octanoate, 15. acetic acid, 16. benzaldehyde, 17. dimethyl sulfoxide, 18. ethyl decanoate, 19. methyl benzoate, 20. phenethyl alcohol
Table 1 Means $( \pm \mathrm{SE})$ of $D$. suzukii captured in traps baited with different treatments in a preliminary field test conducted in Beltsville

\begin{tabular}{llcc}
\hline Treatment & Replicates & $\begin{array}{l}\text { Total no. SWD } \\
\text { captured }\end{array}$ & Mean \pm SE \\
\hline Apple juice blend (7 components) ${ }^{*}$ & 3 & 845 & $281.67 \pm 35.47 a$ \\
Raspberry blend (11 components)** & 3 & 230 & $76.67 \pm 25.17 b$ \\
$2 \mathrm{MB}$ & 3 & 3 & $1.00 \pm 1.00 c$ \\
$3 \mathrm{MB}$ & 3 & 9 & $3.00 \pm 1.00 c$ \\
$2 \mathrm{MB}+3 \mathrm{MB}$ & 3 & 4 & $1.33 \pm 0.58 c$ \\
Blank control & 3 & 1 & $0.33 \pm 0.58 c$ \\
\hline
\end{tabular}

Means in the same column followed by the different letters are significantly different at $\alpha=0.05$ (one-way ANOVA, Ryan-Einot-Gabriel-Welsch $F$ test, $F=120.00, d f=5,12, P<0.001$ ). The numbers of other Drosophila spp. caught in this preliminary test were not counted. *Seven-component blend includes isobutanol (IB), 2-methyl butanol (2 MB), 3-methyl butanol (3 MB), ethyl hexanoate (EH), acetoin (AT), ethyl octanoate (EO), and methyl benzoate (MB) in natural ratios of 4:7:7:0.3:1:0.3:2 (v/v). **Abraham et al. (2015) 
suzukii flies were sent to the Systematic Entomology Laboratory, USDA, ARS, in Beltsville, MD, for taxonomic identification.

Lure dispenser tubes and trap drowning solutions with captured insects were collected and replaced weekly unless otherwise indicated. Flies with morphological characters (approximately $2-3.5 \mathrm{~mm}$ in length and $5-6.5 \mathrm{~mm}$ in wingspan with light yellow or brown body with red eyes, dark unbroken bands across the abdominal segments) were counted in the laboratory. Drosophila suzukii flies were identified with dissecting scopes based on the presence of a dark spot on the wing tips of males and the presence of the serrated ovipositor in females (Walsh et al. 2011). Number of male may not be accurate because young males $(<24 \mathrm{~h})$ sometimes may lack the wing spot (Cini et al. 2012; Hauser 2011). Other Drosophila species collected in the traps were not identified and cited as "other Drosophila spp." in 2015, 2016, and 2017 field tests. Because the blank control traps caught almost nothing, they were not included in the subsequent field tests unless otherwise indicated.

In 2015, three additional treatments, including treatment 14 (two components, including AT and EO in 1:1 ratio), treatment 16 (single component, AT), and treatment 17 (single component, EO), were placed in cotton balls in centrifuge tubes. The rest of compounds, including EtOH $(10 \mathrm{~mL})$ and $\mathrm{AA}(1 \mathrm{~mL})$, were added into drowning water and evaluated as individual attractant or synergistic attractive agent when ever needed (Fig. 4, $N=3-15$ ). In 2016, ACV $\left(300 \mathrm{~mL}\right.$, Essential Everyday ${ }^{\circledR}, 5 \%$ acidity, Shoppers Food and Pharmacy, College Park, MD) and a commercially available Scentry ${ }^{\circledR}$ SWD lure (Great Lakes IPM Inc., Vestaburg, MI) were used as standard lures for SWD attraction activity comparison. In addition, the amount of AA in the drowning solution in each trap was increased from 1 to $15 \mathrm{~mL}$ to reach the same acidity (5\%) with ACV. In addition, ethyl acetate (EA) $(5 \mathrm{~mL})$ and phenethyl alcohol (PE) $(1 \mathrm{~mL})$ were added to the drowning solution and evaluated as individual attractants or synergistic attractive agents in 2016 (Fig. 5a-c, $N=6$ ). Furthermore, additional tests using quinary blend $(\mathrm{AT}+\mathrm{EO}+\mathrm{EA}+\mathrm{AA}+\mathrm{PE})$ were conducted at the blueberry field in Butler's Orchard, MD (Fig. 5d, $N=9$ ).

In 2017, the controlled release rate dispenser of quinary blend $(\mathrm{AT}+\mathrm{EO}+\mathrm{EA}+\mathrm{AA}+\mathrm{PE})$ was formulated by ChemTica International, S.A. (Heredia Province, Santa Rosa, Costa Rica) and compared with ACV, Sentry lure, and laboratory-made quinary blend formulation (in polypropylene flex micro-centrifuge tube) at the blueberry field during the middle of blueberry field season (June 29-July 5 at the Butler's Orchard, MD (Table 3, $N=12$ ).

\section{Laboratory behavioral bioassay}

Given that the apple juice seven-component blend (treatment 1) was attractive to $D$. suzukii in the preliminary field tests conducted in 2014 (Table 1), a laboratory dual-choice behavioral bioassay was designed to refine this blend in order to identify the key attractive component and examine the other factors, which may have influence to SWD attraction. A complete test apparatus consisted of a plastic cup containing two trap tubes with two lure vials, and a water wick (Fig. 2). The plastic cups were clear, cylindrical polypropylene food containers $(946 \mathrm{~mL}$, diameter $114 \mathrm{~mm}$, height $127 \mathrm{~mm}$, Paper Mart, CA, USA). The lid of each cup had an 80-mmdiameter circular hole cut in it, which was covered with a nylon mesh (no-thrips insect screen, mesh size: $81 \times 81$, Bioquip, CA, USA) to provide ventilation while retaining flies. The polystyrene trap tubes $(95 \mathrm{~mm} \times 28.5 \mathrm{~mm}$, same as those used in insect rearing) were labeled " $\mathrm{T}$ " for treatment and "B" for blank control, respectively, and were then given the appropriate lure. Lures were either tested chemical/chemical blends (unless otherwise indicated, $20 \mu \mathrm{l}$ pure individual or blend) loaded onto a small cotton ball held within a small polyethylene lure vial $(26 \mathrm{~mm} \times 8 \mathrm{~mm} \times 1.5 \mathrm{~mm}$ thickness, Just Plastic Ltd., Norwich, UK) or blank controls of plain cotton or cotton with solvent (in dose response trials) held in the same type of small lure vial. Each lure vial (open lid) was placed vertically within its trap tube, and then, the top of each trap tube was sealed with Parafilm ${ }^{\circledR}$ (Pechiney Plastics Packaging Inc., Menasha, WI, USA) leaving only a 4-mm-diameter hole in the center to provide an entrance for attracted flies. Each pair of loaded trap tubes was then

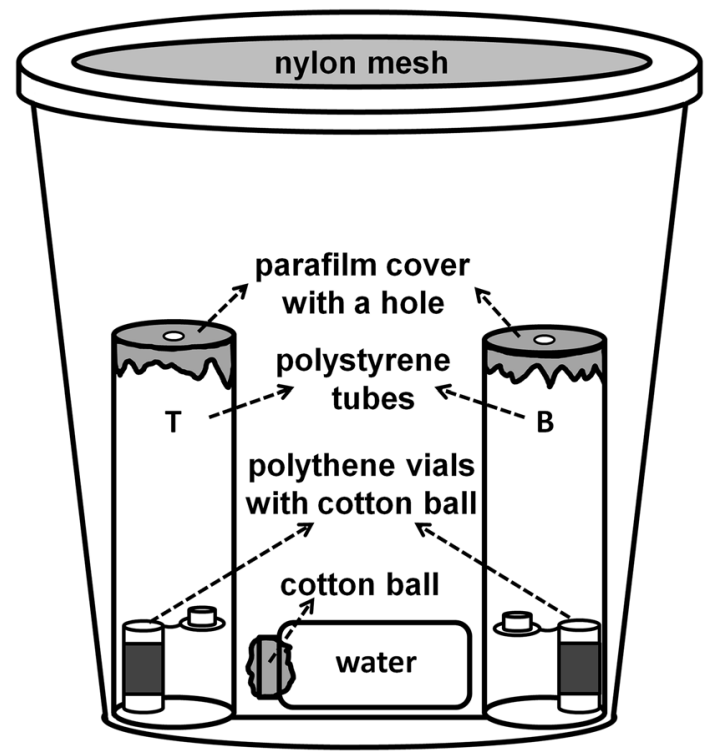

Fig. 2 Laboratory dual-choice bioassay apparatus used in experiment. $T$ treatment tube, $B$ blank control tube 
placed vertically on opposite sides of the test cups with the entrance holes facing upwards. A water wick consisting of a glass scintillation vial (20 mL, VWR, PA, USA) filled with deionized water and plugged with a cotton ball was laid on the bottom of the plastic cup to serve as a water source for the flies during the experiment (Fig. 2). Flies were immobilized using a $\mathrm{CO}_{2}$ stream, and ten were transferred into each testing apparatus. The fully assembled test apparatus cups were then covered with their ventilated lids.

In order to determine the optimized amount of chemical to be used in dual-choice laboratory experiments, a dose response experiment (treatment 1 vs. blank control) was first carried out $(N=3$, Fig. 3a, c). Since ethanol $(\mathrm{EtOH})$ and acetic acid (AA) have been previously reported as the major SWD attractants (Cha et al. 2014), they were examined individually and in different combinations with treatment 1 ( $50 \mu$ l total volume, $N=5$, Fig. $3 d$ ). Component exclusion test was then performed to identify the key attractive components $(N=3-11$, Fig. 3b). Individual and different combinations of three final attractive candidates were evaluated $(N=3-8$, Fig. 3e). Finally, a dose response test of the most attractive component was assessed $(N=5$, Fig. 3f). All assays were conducted in a fume hood $(120 \mathrm{~cm}$ length $\times 60$ $\mathrm{cm}$ width $\times 70 \mathrm{~cm}$ height) using new lures and young flies $(N=3-11,2-8$ days post-eclosion, directly from cornmeal diet rearing tubes with unknown mating status) under $25^{\circ} \mathrm{C}$, $60 \% \mathrm{RH}$, and a 16:8 h (L/D) photoperiod. Mixed-sex $D$. suzukii were used in bioassays unless stated otherwise, and all flies within and outside treatment and control trap tubes were counted and sexed after $48 \mathrm{~h}$.

\section{Lure release rate}

Release rates of three different kinds of attractants, including acetoin (AT), ethyl octanoate (EO), and a blend of AT and $\mathrm{EO}$ (ratio = 1:1), were measured under controlled conditions in a laboratory fume hood. Each attractant $(1 \mathrm{~mL})$ was loaded onto cotton balls held in open micro-centrifuge tubes (1.5 mL, VWR International, Radnor, PA). Fifteen of these micro-centrifuge tubes $(N=5)$ were suspended on hooks in a fume hood (temperature $20-25{ }^{\circ} \mathrm{C}$, face velocity 129 feet/min). Each tube was weighed using an Ohaus GA110 analytical electronic balance (Pine Brook, NJ) every 24 or $72 \mathrm{~h}$ (weekend), and the amount of attractant residue was calculated and recorded over a period of 2 weeks.

\section{Statistical analyses}

Data from bioassays that evaluated the SWD response to treatment trap tubes versus control trap tubes in dualchoice tests were converted to percentages and analyzed using $G$ tests (Microsoft Office Excel 2007) (Sokal and Rohlf 1995) with the null hypothesis that $D$. suzukii would be in a 1:1 distribution (50\% of choices of each trap tube). The mean number of $D$. suzukii in different treatment trap tubes for laboratory assays (captures/2 days) and that in different treatment traps (captures/week) in field trials were compared by one-way analysis of variance (ANOVA) followed by Ryan-Einot-Gabriel-Welsch $F$ test (SPSS 10.0 for Windows) (George and Mallery 2002). Some field data ranges showed a strong positively skewed distribution (skewness/standard error of skewness $>1.96$; therefore, the null hypothesis of normality was rejected); therefore, square root transformations were performed to remedy non-normality prior to statistical analyses. All statistical comparisons were considered for significance at $\alpha=0.05$. Attractants release rate data (decline of different kinds of attractants from open micro-centrifuge tubes) were fitted with exponential trendline (Microsoft Office Excel 2007), from which the half-life times $\left(t_{1 / 2}\right)$ of the lures were calculated.

\section{Results}

\section{Identification of the volatile compounds from apple juices}

GC-MS analyses of apple juice headspace extracts revealed that several volatile compounds were present in comparable amounts in both fresh and fermented samples (Fig. 1). Since it has been reported that the yeast microbe volatiles could mediate Drosophila flies attraction (Becher et al. 2010, 2012), special attention was given to the compounds produced and/or enriched during the fermentation process. We found that consistently higher levels $(>10 \times$ fold ratio relative to fresh apple juice volatile extract) of five compounds were associated with fermented apple juice volatile extract (Fig. 1, top trace). The compounds were identified as isobutanol (IB, peak 2), 2- and 3-methyl butanol (2 and $3 \mathrm{MB}$, peak 6), 3-hydroxy-2-butanone ([also called as acetoin (AT)], peak 10), and acetic acid (AA, peak 15) in an approximate ratio of 4:7:7:1:8 (v/v), respectively. Four compounds, including ethyl hexanoate (EH, peak 8), ethyl octanoate (EO, peak 14), ethyl decanoate (ED, peak 18), and methyl benzoate (MB, peak 19), were only produced by the fermentation in an approximate ratio of 0.3:0.3:0.2:2(v/v), respectively, and absent in fresh apple juice volatile sample (Fig. 1, bottom trace). Compound, phenethyl alcohol (PE, peak 20), was also a volatile component enriched by fermentation. Two additional compounds, ethyl acetate (EA) and ethanol (EtOH), were detected as major headspace volatile components by SPME sampling method from both fresh and fermented apple juices. They were masked by solvent peak 


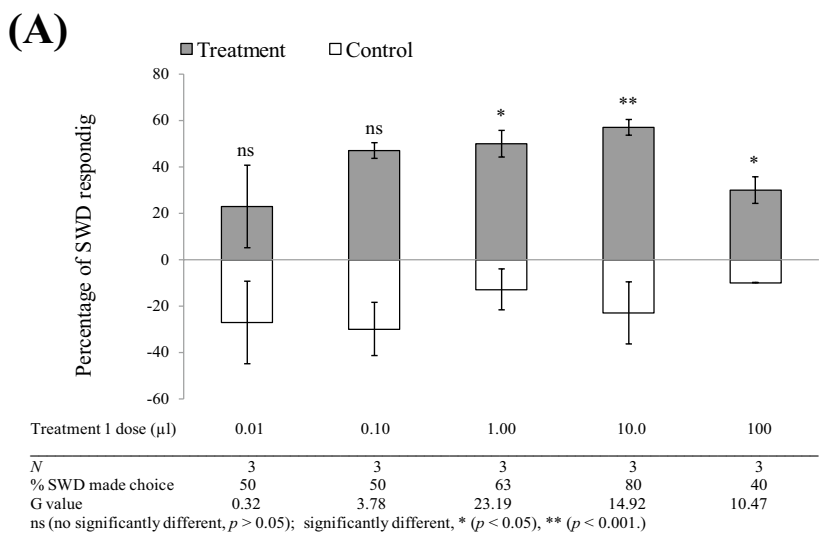

(C)

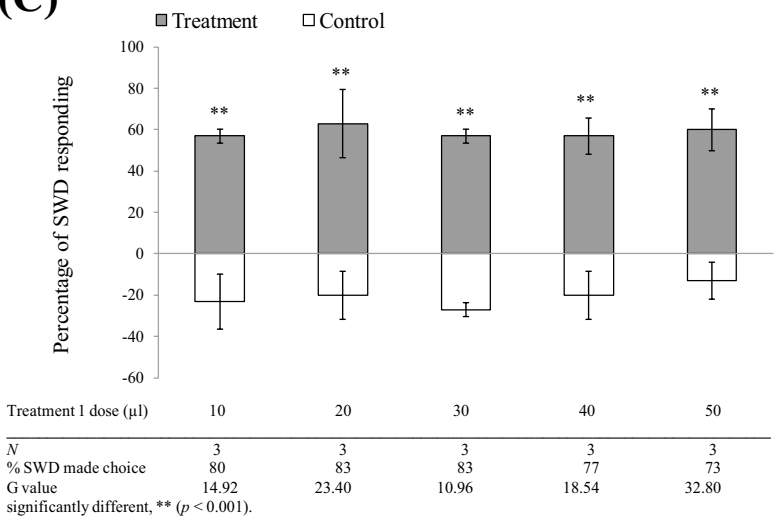

(E)

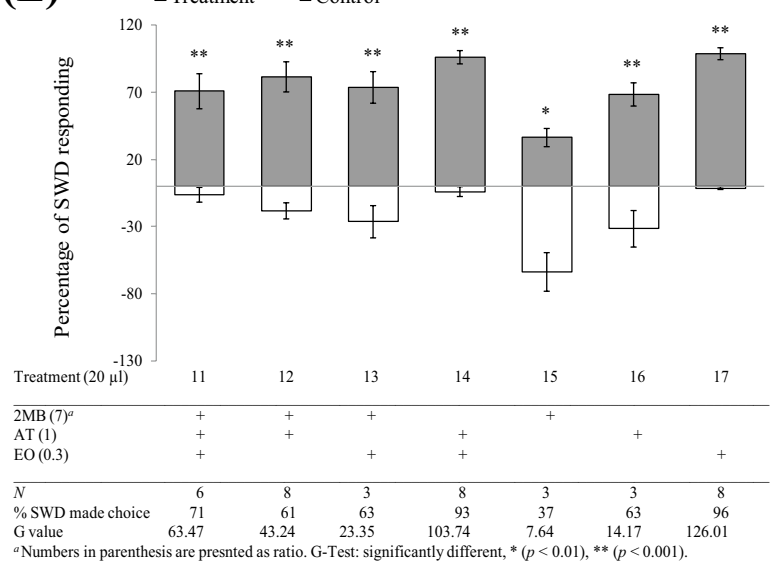

Fig. 3 Behavioral responses (mean $\pm \mathrm{SE}$ ) of adult D. suzukii (male and female) against blank control in laboratory dual-choice bioassay to: a treatment 1 (seven components, $0.01-100 \mu$ d dose response); c treatment 1 (seven components, $10-50 \mu$ dose response); $\mathbf{d}$ treatment 1 (seven components, effects of ethanol, acetic acid, and $10 \%$ acetic acid/ethanol solution); b treatment 1 (seven components), 2-8 (six components), 9 (five components), and 10 (four components), and 11
(B)



(D)

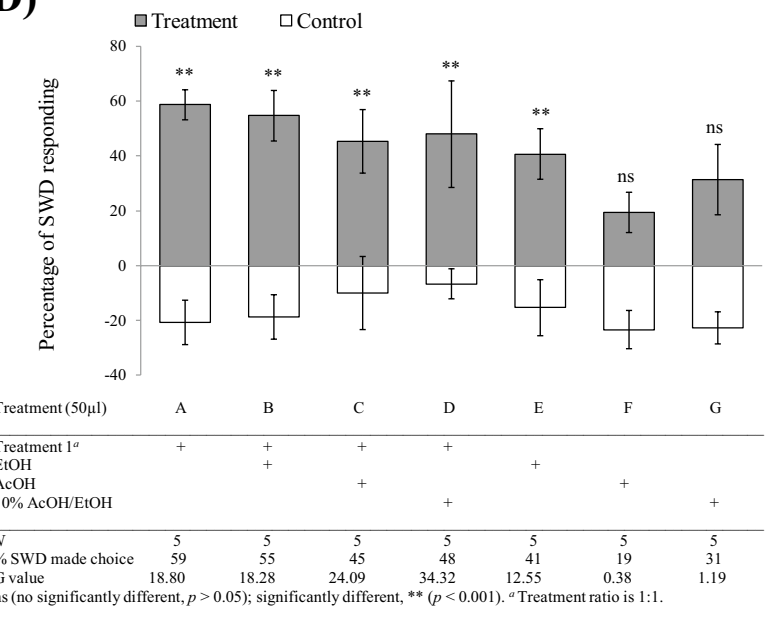

(F)

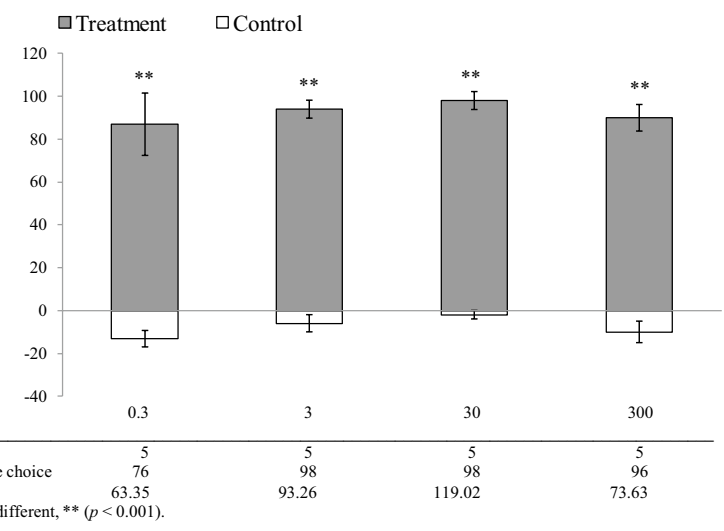

(three components); e treatments 11 (three components), 12, 13, 14 (two components), and 15, 16, 17 (single component); $\mathbf{f}$ treatment 17 (single component, EO, 0.3-300 $\mu \mathrm{l}$ dose response). Data are average percent of flies choosing one of the tubes $48 \mathrm{~h}$ after release. Experiments were replicated 3-11 times with ten flies each. Trapping data were analyzed by $G$ tests: ns (not significantly different, $P>0.05$ ), significantly different, $* P<0.05, * * P<0.001$ 
in conventional GC-MS analyses of apple juice headspace extracts.

\section{Preliminary field test}

During a 1-week preliminary field test from October 17-24, 2014, at Beltsville, MD, a total of 845 adult D. suzukii were captured in all traps baited with apple juice seven-component blend (treatment 1), 230 D. suzukii were trapped in all traps baited with raspberry 11-component blend (Abraham et al. 2015), and only 17 D. suzukii were caught in all traps baited with 2, 3, and $2 \mathrm{MB}+3 \mathrm{MB}$ blend (the most abundant components in fermented apple juice) and blank control traps. The numbers of other Drosophila spp. caught in traps were not counted at this time. Our data demonstrated that traps baited with treatment 1 captured significantly more male and female $D$. suzukii than traps baited with other treatments and blank controls $(F=120.00 ; d f=5,12 ; P<0.001)$ (Table 1), indicating that the apple juice seven-component blend (treatment 1) contained some critical attractive components for attracting $D$. suzukii.

\section{Laboratory dual-choice bioassays}

\section{Dose response of apple juice seven-component blend}

The doses above $1 \mu \mathrm{l}(\sim 1 \mathrm{mg})$ were found to be attractive $(0.01,0.1,1,10$, and $100 \mu \mathrm{l})$ when treatment 1 blend was tested (0.01: $G=0.32, d f=1, P=0.57 ; 0.1: G=0.3 .78$, $d f=1, P=0.052 ; 1: G=0.23 .19, d f=1, P=1.47 \mathrm{E}-6$; 10: $G=14.92, d f=1, P=1.2 \mathrm{E}-4 ; 100: G=10.47, d f=1$, $P=0.012$ ) (Fig. 3a). An additional dose response experiment, using $10,20,30,40$, and $50 \mu \mathrm{l}$, was conducted. Similar activities were obtained and no significant differences were found in this dose range (10: $G=14.92, d f=1$, $P=1.12 \mathrm{E}-04 ; 20: G=23.40, d f=1, P=1.32 \mathrm{E}-06 ; 30$ : $G=10.96, d f=1, P=9.34 \mathrm{E}-04 ; 40: G=18.54, d f=1$, $P=1.7 \mathrm{E}-05$; 50: $G=32.80, d f=1, P=1.02 \mathrm{E}-08$ ) (Fig. 3c). Therefore, unless otherwise indicated, an amount of $20 \mu \mathrm{l}$ $(\sim 20 \mathrm{mg})$ for each chemical or blend was used as the standard dose in all other experiments. Because the sex ratios of D. suzukii were found to be close to 1:1 in the entire trap and control tubes, sex ratio determination was omitted in subsequent laboratory experiments.

\section{Effects of ethanol and acetic acid to apple juice seven-component blend}

Our results indicated that treatment 1 (A, seven components) and EtOH alone (E), as well as combinations of treatment 1 with EtOH (B), AA (C), 10\% AA in EtOH (D), were significantly more attractive compared to a blank control (A: $G=18.80, d f=1, P=1.50 \mathrm{E}-05$; B: $G=18.28$, $d f=1, P=1.90 \mathrm{E}-05 ; \mathrm{C}: G=24.09, d f=1, P=9.19 \mathrm{E}-07$; D: $G=34.32, d f=1, P=4.68 \mathrm{E}-09$; E: $G=12.55, d f=1$, $P=3.97 \mathrm{E}-04$; F: $G=0.38, P=0.54$; G: $G=1.19, d f=1$, $P=0.28$ ) (Fig. 3c), while AA (F) and 10\% AA/EtOH (G) alone were not attractive, and no synergistic effects were observed when treatment 1 blend was combined with $\mathrm{EtOH}$ (B), AA (C), and 10\% AA/EtOH (D) treatments (Fig. 3d).

\section{Determination of key components from the apple juice seven-component blend}

To determine the key attractive components from the seven-component synthetic blend (treatment 1 ), seven six-component blends (treatments 2-8) were prepared by eliminating one component from treatment 1 and one five-, four-, and three-component (treatments 9-11) blends were prepared by eliminating two, three, and four components, respectively, and compared to treatment 1 ( $1: G=47.55$, $d f=1, P=5.64 \mathrm{E}-12 ; 2: G=69.33, d f=1, P=8.34 \mathrm{E}-17$; 3: $G=38.55, d f=1, P=5.34 \mathrm{E}-10 ; 4: G=93.24, d f=1$, $P=4.76 \mathrm{E}-22 ; 5: G=18.20, d f=1, P=2.00 \mathrm{E}-05$; 6: $G=54.09, P=1.92 \mathrm{E}-13 ; 7: G=38.55, d f=1$, $P=5.34 \mathrm{E}-10 ; 8: G=87.90, P=6.88 \mathrm{E}-21 ; 9: G=30.77$, $d f=1, P=2.90 \mathrm{E}-08 ; 10: G=63.93, d f=1, P=8.34 \mathrm{E}-17$; 11: $G=98.92, d f=1, P=2.62 \mathrm{E}-23$ ) (Fig. 3b). Although no significant activity reduction was observed in this component exclusion experiment, the three-component blend (treatment 11) showed the same attractive capacity as the complete treatment 1 blend, indicating that this blend ( $2 \mathrm{MB}$, AT, and EO) may contain the key attractive components (Fig. 3b). Consequently, this three-component blend (treatment 11) was further tested as two-component blends and as individual components. The results clearly demonstrated that an individual component, $2 \mathrm{MB}$ (treatment 15), exhibited repellent effect. When $2 \mathrm{MB}$ was used alone, blank control tubes trapped significantly more $D$. suzukii than the $2 \mathrm{MB}$ treatment tubes (treatment 15, Fig. 3e). In addition, $2 \mathrm{MB}$ elicited the lowest percentage response from D. suzukii. Compared with the attractant component ethyl benzoate (EO, treatment 17 ) in which $96 \%$ of $D$. suzukii made choice, only $37 \%$ of $D$. suzukii made choice when 2 MB was present (treatment 15, Fig. 3e). However, the treatments 16 (AT) and 17 (EO) individually elicited significant attraction to D. suzukii and the EO elicited the higher percentage response $(96 \%)$ than that of AT $(63 \%)$ from $D$. suzukii (11: $G=63.47, d f=1, P=1.63 \mathrm{E}-15 ; 12: G=43.24$, $d f=1, P=4.85 \mathrm{E}-11 ; 13: G=23.35, d f=1, P=1.35 \mathrm{E}-06$; 14: $G=103.74, d f=1, P=2.31 \mathrm{E}-24 ; 16: G=14.17, d f=1$, $P=1.70 \mathrm{E}-03 ; 17: G=126.01, d f=1, P=2.98 \mathrm{E}-29)$ (Fig. 3e). Different doses of EO (from 0.3 to $300 \mu$ levels) were examined to determine whether the amount of this compound loaded on the bait might affect the biological 
activity. Significantly attractive activities were observed for all doses tested in this assay, even at the lowest dose $0.3 \mu \mathrm{l}$ $(0.3 \mathrm{mg})(0.3: G=63.47, d f=1, P=4.77 \mathrm{E}-15 ; 3: G=93.26$, $d f=1, P=4.64 \mathrm{E}-22 ; 30: G=119.02, d f=1, P=1.04 \mathrm{E}-27$; 300: $G=73.63, d f=1, P=9.50 \mathrm{E}-18$ ) (Fig. 3f).

\section{Activity comparison of ethyl octanoate with raspberry extract}

Effectiveness of treatment 17 (EO, single component) was compared with treatment 1 (seven-component blend), treatment 11 (three-component blend), treatment 14 (two-component blend), and raspberry extract ( $50 \mu$ loading). Trap tubes baited with the single component, EO, captured significantly more $D$. suzukii than trap tubes baited with treatment 1 (seven components) and treatment 11 (three components). In addition, EO (treatment 17) was also as attractive as treatment 14 (two components, composed of EO and AT) and natural raspberry extract $(F=10.167, d f=4,20 ; P<0.001)$ (Table 2).

\section{Additional field tests}

\section{5}

Given that the single component, ethyl octanoate (EO, treatment 17), was the most attractive component for D. suzukii in laboratory bioassays (Fig. 3e, f), it was tested at Beltsville, Agricultural Research Center, MD, in the late fall during October 14 to November 18, 2015. Interestingly, EO alone did not show any activity at all, while the AT (treatment 16) was significantly more attractive than $\mathrm{EO}(F=15.78$, $d f=4,45, P<0.0001$ ) (Fig. 4a). In addition, the attraction of AT was significantly enhanced when it was combined

Table 2 Means $( \pm \mathrm{SE})$ of $D$. suzukii captured in tubes baited with different treatments against blank control in dual-choice laboratory bioassays

\begin{tabular}{lllll}
\hline Treatment & Replicates & $\begin{array}{l}\text { Total } \\
\text { no. } \\
\text { tested }\end{array}$ & $\begin{array}{l}\text { Total no. } \\
\text { captured }\end{array}$ & Mean \pm SE \\
& & 50 & 23 & $4.60 \pm 2.19 c$ \\
1 (7 components)* & 5 & 50 & 26 & $5.20 \pm 2.28 b c$ \\
11 (2 MB + AT+EO) & 5 & & & \\
$14($ AT + EO) & 5 & 50 & 47 & $9.40 \pm 0.55 a$ \\
17 (EO) & 5 & 50 & 49 & $9.80 \pm 0.45 a$ \\
Raspberry extract & 5 & 50 & 38 & $7.60 \pm 1.81 a b$ \\
\hline
\end{tabular}

Means in the same column followed by the different letters are significantly different at $\alpha=0.05$ (one-way ANOVA, Ryan-Einot-GabrielWelsch $F$ test, $F=10.167, d f=4,20, p<0.001)$. *Seven-component blend includes isobutanol (IB), 2-methyl butanol (2 MB), 3-methyl butanol (3 MB), ethyl hexanoate (EH), acetoin (AT), ethyl octanoate (EO), and methyl benzoate (MB) in natural ratios of 4:7:7:0.3:1:0.3:2 $(\mathrm{v} / \mathrm{v})$ with (AA), but this enhanced effect was not observed for nontarget Drosophila species (for SWD: $F=15.78, d f=4,45$, $P<0.0001$; for other Drosophila spp.: $F=9.71, d f=4,45$, $P<0.0001$ ) (Fig. 4a). Furthermore, activity of EtOH was evaluated in the field. The same result as laboratory bioassay was obtained; EtOH alone did not show significant activity; instead, it attracted significantly more nontarget Drosophila when it was combined with the AT + AA blend (for other Drosophila spp., $F=9.71, d f=4,45, P<0.0001$ ) (Fig. 4a). The synergistic effect of AA to AT was confirmed in a later test. Significantly more $D$. suzukii were caught in the trap baited with AT when AA was added to the drowning solution than the traps baited with AT or AA alone $(F=12.89, d f=2,42 ; P<0.001)$ (Fig. 4b). Although EO alone did not show any activity in the field (Fig. 4a), it significantly enhanced the attraction of AT $(\mathrm{AT} / \mathrm{EO}=1: 1)$, but did not affect the trap catch of other Drosophila species (for SWD: $F=7.45, d f=2,6, P<0.05$; for other Drosophila spp.: $F=1.49, d f=2,6, P=0.30$ ) (Fig. 4c). No synergistic effect was observed when EO was combined with AA.

\section{6}

Our field data demonstrated that a mixture of EA and AA was moderately attractive to $D$. suzukii, but it was less attractive than a ternary blend (AT +EO+AA). However, when EA was added to this ternary blend to form a quaternary blend (AT + EO+EA + AA), attraction to D. suzukii was significantly increased, while nontarget Drosophila attraction was not (for male: $F=63.77, \mathrm{df}=2,6, P<0.0001$; for female: $F=16.25, d f=2,6, P<0.01$; for other Drosophila spp.: $F=7.06, d f=2,6, P<0.05$; for total SWD: $F=52.24, d f=2,6, P<0.001)$ (Fig. $4 \mathrm{~d}$ ). The quaternary blend $(\mathrm{AT}+\mathrm{EO}+\mathrm{EA}+\mathrm{AA})$ was also significantly more attractive to $D$. suzukii than the widely used ACV and commercially available Scentry ${ }^{\circledR}$ SWD lure under field conditions (for male: $F=34.57, d f=4,25, P<0.0001$; for female: $F=39.94, d f=4,25, P<0.0001$; for other Drosophila spp.: $F=26.01, d f=4,25, P<0.0001$; for total SWD: $F=37.16$, $d f=4,25, P<0.0001$ ) (Fig. 5a). However, like AA and EtOH, EA itself was not attractive in the field (Fig. 5a). The population of SWD was unusually high in the middle of November in Beltsville, MD. During a 1-week trapping period, a total of $\sim 77,600 \mathrm{D}$. suzukii were captured by traps baited with the quaternary blend $(\mathrm{AT}+\mathrm{EO}+\mathrm{EA}+\mathrm{AA})$, yielding an average of 13,000 D. suzukii per trap. During the same period, a total of $\sim 28,500 \mathrm{D}$. suzukii were captured by traps baited with ACV, yielding an average of $4700 D$. suzukii per trap and a total of $\sim 18,800 \mathrm{D}$. suzukii were captured by traps baited with Scentry ${ }^{\circledR}$ lure, yielding an average of 3100 D. suzukii per trap. In addition, traps baited with the quaternary blend $(\mathrm{AT}+\mathrm{EO}+\mathrm{EA}+\mathrm{AA})$ captured much less nontarget Drosophila than ACV and Scentry ${ }^{\circledR}$ 


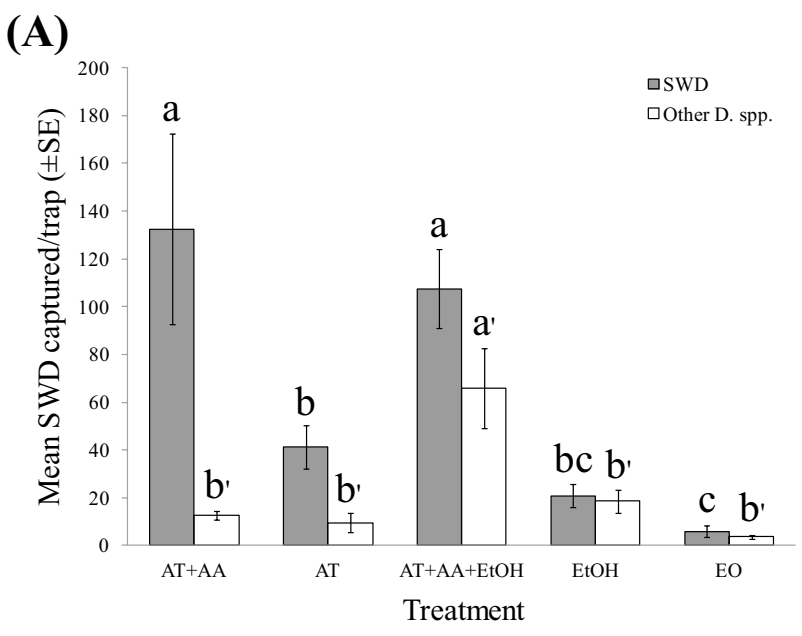

(C)

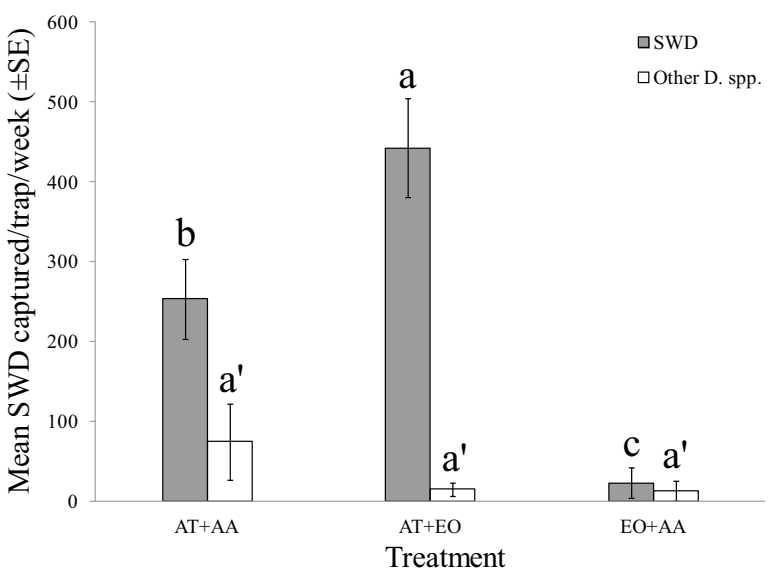

Fig. 4 Mean $( \pm \mathrm{SE})$ trap catches of D. suzukii and other Drosophila spp. in traps baited with different combinations of acetoin (AT), acetic acid (AA), ethyl octanoate (EO), ethanol (EtOH), and ethyl acetate (EA) deployed at the Beltsville Agricultural Center, Maryland. Identically colored bars with the different letters and superscripts above them are significantly different at $\alpha=0.05$ (one-way ANOVA, square root transformed, Ryan-Einot-Gabriel-Welsch $F$ test). a Oct. 14-Oct. 21, $2015(N=10, d f=4,45$; for SWD: $F=15.78 ; P<0.0001$;

lures, thus demonstrating its higher selectivity for D. suzukii attraction (SWD/other Drosophila spp. ratio: quaternary blend $=31.95$, ACV $=15.90$, Scentry $=10.16)($ Fig. 5a). The synergistic effect of EA was further confirmed in a subsequent 4-week field test (total captures, lures, and contents were not changed weekly). The newly formed quaternary blend $(\mathrm{AT}+\mathrm{EO}+\mathrm{EA}+\mathrm{AA})$ attracted significantly more $D$. suzukii than the ternary blend (AT + EO + AA), ACV, and Scentry ${ }^{\circledR}$ lures, but did not affect attraction of other Drosophila species (for male: $F=10.38, d f=3,20, P<0.001$; for female: $F=6.36, d f=3,20, P<0.01$; for other Drosophila spp.: $F=1.12, d f=3,20, P=0.366$; for total SWD: $F=8.86$, $d f=3,20, P<0.001$ ) (Fig. 5b). Again, the quaternary blend

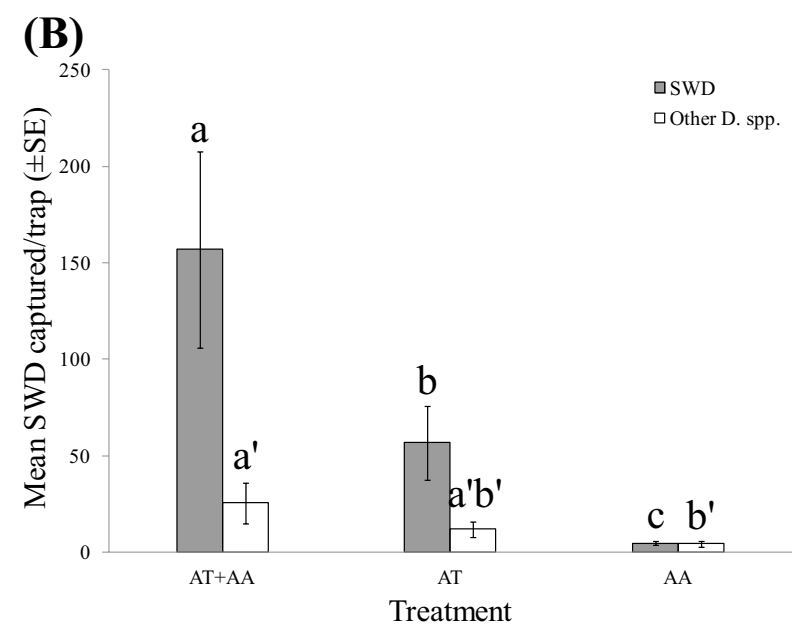

(D)

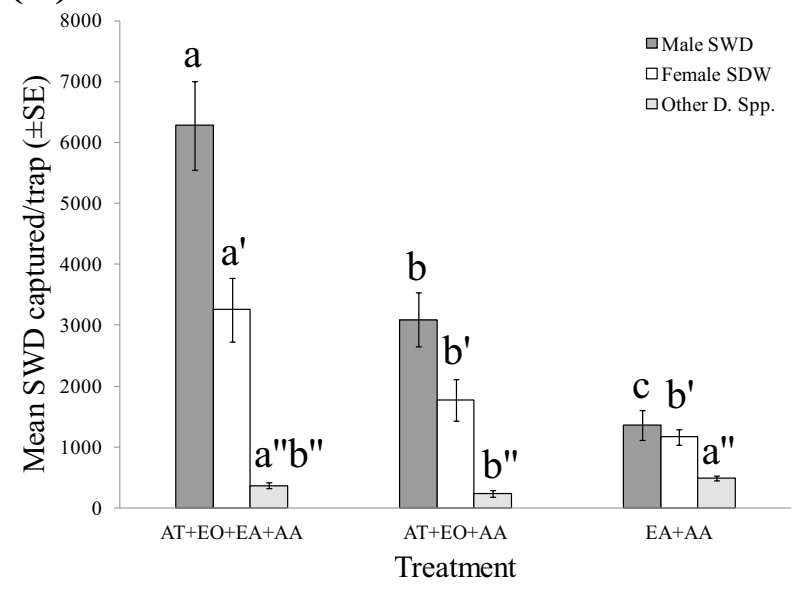

for other Drosophila spp.: $F=9.71, P<0.0001$ ); b Oct. $21-$ Oct. 28 $(N=15, d f=2,42$; for SWD: $F=12.89, P<0.0001$; for other Drosophila spp.: $F=5.93, P<0.01)$; c Oct. $28-$ Nov. 18, 2015 for 3 weeks $(N=3, d f=2,6$; for SWD: $F=7.45, P<0.05$; for other Drosophila spp.: $F=1.49, P=0.30)$; d Nov. 8-Nov. 15, $2016(N=3, d f=2,6$; for male: $F=63.77, P<0.0001$; for female: $F=16.25, P<0.01$; for other Drosophila spp.: $F=7.06, P<0.05)$

$(\mathrm{AT}+\mathrm{EO}+\mathrm{EA}+\mathrm{AA})$ demonstrated the highest SWD/ other Drosophila spp. ratio during this period (quaternary blend $=11.43, \mathrm{ACV}=9.29$, Scentry $=7.55$ ).

Fermented apple juice also produced significantly more PE (compound 20, Fig. 1) compared to fresh apple juice. When tested in the field, PE, like EA, did not show any activity for $D$. suzukii attraction compared to acetoin AT (for male: $F=9.09, d f=2,15, P<0.01$; for female: $F=11.55$, $d f=2,15, P<0.001$; for other Drosophila spp.: $F=18.61$, $d f=2,15, P<0.0001$ ) (Fig. $5 \mathrm{c}$ ). However, by adding the PE into the quaternary blend (AT $+\mathrm{EO}+\mathrm{EA}+\mathrm{AA})$ to form a quinary blend $(\mathrm{AT}+\mathrm{EO}+\mathrm{EA}+\mathrm{AA}+\mathrm{PE})$, it significantly enhanced SWD attraction, but did not affect trap catch of 
(A)

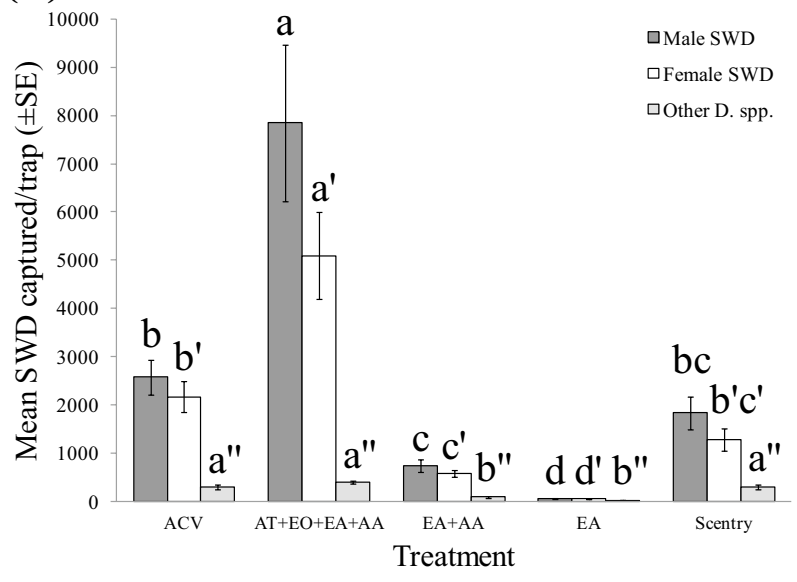

(C)

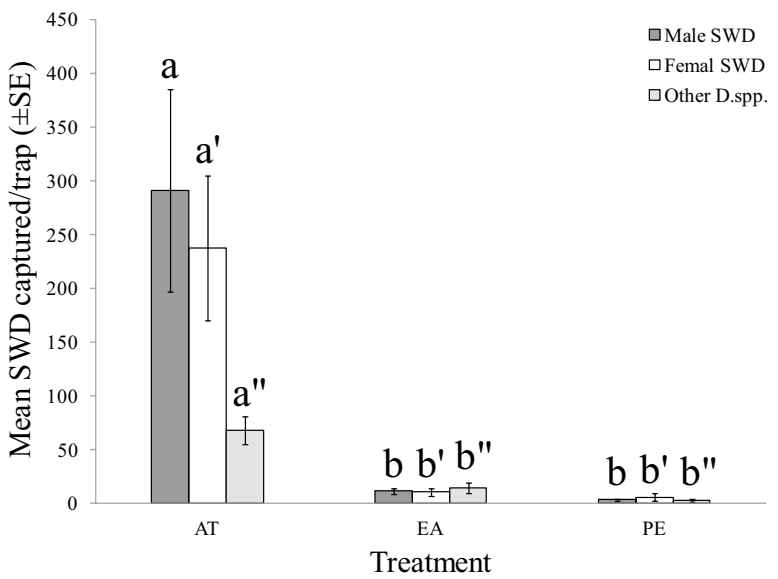

Fig. 5 Mean $( \pm \mathrm{SE})$ trap catches of D. suzukii and other Drosophila spp. in traps baited with different combinations of acetoin (AT), ethyl octanoate (EO), ethyl acetate (EA), acetic acid (AA), and phenethyl alcohol (PE), as well as apple cider vinegar (ACV) and commercial SWD lure from Scentry ${ }^{\circledR}$ (Scentry) deployed at the Beltsville Agricultural Center and at the Butler's Orchard blueberry field, Maryland. Identically colored bars with the different letters and superscripts above them are significantly different at $\alpha=0.05$ (one-way ANOVA, square root transformed, Ryan-Einot-GabrielWelsch $F$ test). a November 10-18, 2016, at the Beltsville Agricultural Center $(N=6, d f=4,25$; for male: $F=34.57, P<0.0001$; for female: $F=39.94, P<0.0001$; for other Drosophila spp.: $F=26.01$,
(B)

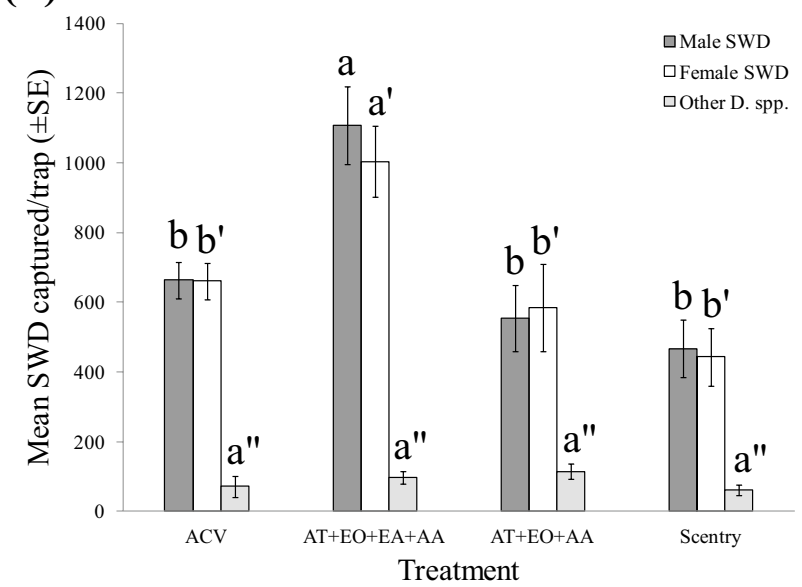

(D)

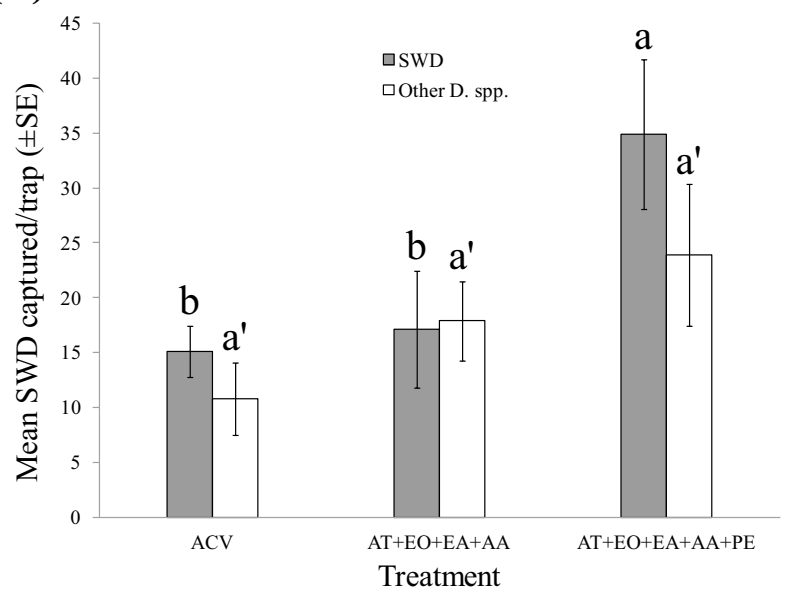

$P<0.0001)$; b November 25-December 22, 2016, for 27 days at the Beltsville Agricultural Center (total trap captures, lures, and contents were not changed and collected weekly) $(N=6, d f=3,20$; for male: $F=10.38, P<0.001$; for female: $F=6.36, P<0.01$; for other Drosophila spp.: $F=1.12, P=0.366$ ); c December 2-22, 2016, for 20 days at the Beltsville Agricultural Center (total trap captures, lures, and contents were not changed and collected weekly) $(N=6, d f=2,15$; for male: $F=9.09, P<0.01$; for female: $F=11.55, P<0.001$; for other Drosophila spp.: $F=18.61, P<0.0001)$; d November 30-December 7, 2016, at the Butler's Orchard blueberry field $(N=9, d f=2,24$; for SWD: $F=4.47, P<0.05$; for other Drosophila spp.: $F=1.96$, $P=0.163)$

Table 3 Means $( \pm \mathrm{SE})$ of $D$. suzukii and other arthropods captured per trap at the Butler's Orchard blueberry field

\begin{tabular}{llrllrr}
\hline Treatment & \multicolumn{1}{l}{ SWD } & \multicolumn{1}{c}{ SWD } & Other Drosophila spp. & Other Diptera & Other arthropods & SWD \% \\
\hline Control & $0 b$ & $0.08 \pm 0.08 b$ & $0 b$ & $0.83 \pm 0.21 b$ & $4.25 \pm 0.69 a$ & $1.04 \pm 1.04 d$ \\
ACV & $0.75 \pm 0.35 b$ & $1.67 \pm 0.41 b$ & $11.00 \pm 1.82 b$ & $10.25 \pm 2.04 a$ & $13.25 \pm 3.69 a$ & $6.40 \pm 1.62 d$ \\
Scentry & $15.25 \pm 3.05 a$ & $41.00 \pm 9.02 a$ & $116.08 \pm 21.21 a$ & $7.00 \pm 1.43 a$ & $21.17 \pm 18.48 a$ & $26.84 \pm 2.75 c$ \\
ChemTica & $4.67 \pm 1.18 b$ & $18.08 \pm 4.19 b$ & $2.75 \pm 0.99 b$ & $0.17 \pm 0.11 b$ & $3.00 \pm 1.54 a$ & $72.30 \pm 5.51 a$ \\
Quinary blend & $1.17 \pm 0.36 b$ & $6.58 \pm 1.98 b$ & $6.25 \pm 1.13 b$ & $0 b$ & $1.67 \pm 0.50 a$ & $47.24 \pm 4.27 b$ \\
\hline
\end{tabular}

Means in the same column followed by the different letters are significantly different at $\alpha=0.05$ (one-way ANOVA, Ryan-Einot-GabrielWelsch $F$ test. $N=12, d f=4,55$ ). For male SWD: $F=18.38, P<0.0001$; for female SWD: $F=13.89, P<0.0001$; for other Drosophila spp.: $F=27.27, P<0.0001$; for other Diptera, $F=17.62 ; P<0.0001$; for other arthropods, $F=1.60 ; P=0.19$; for SWD $\%$ : $F=72.77, P<0.0001$. No pesticide sprayed during trapping experiment 
nontarget Drosophila at the Butler's Orchard blueberry field (for SWD: $F=4.47, d f=2,24, P<0.05$; for other Drosophila spp.: $F=1.96, d f=2,24, P=0.163$ ) (Fig. $5 \mathrm{~d}$ ).

\section{7}

Activity of a quinary blend (AT + EO + EA + AA + PE) was further confirmed at the Butler's Orchard blueberry field during the middle of blueberry field season. ChemTica controlled release rate dispenser and our laboratorymade quinary blend formulation caught $\sim 72$ and $\sim 47 \%$ D. suzukii, respectively, which were significantly more selective for D. suzukii attraction than Scentry lure ( 27\%) and ACV $(\sim 6 \%)$ formulations (for male SWD: $F=18.38$, $d f=4,55, P<0.0001$; for female SWD: $F=13.89, d f=4,55$, $P<0.0001$; for other Drosophila spp.: $F=27.27, d f=4,55$, $P<0.0001$; for other Diptera: $F=17.62, d f=4,55$, $P<0.0001$, for other arthropods: $F=1.60, d f=4,55$, $P=0.19$; for SWD $\%: F=72.77, d f=4,55, P<0.0001$ ), although Scentry lure caught significantly more $D$. suzukii (Table 3).

\section{Release rates of major attractants}

Our laboratory release rate study demonstrated that the major attractant AT, close-range attractant $\mathrm{EO}$, and a blend of AT and EO (ratio = 1:1) were desorbed from micro-centrifuge tube dispensers following first-order kinetics (Fig. 6, for AT, $r^{2}=0.9046$; for blend of AT and EO, $r^{2}=0.9373$; for
$\left.\mathrm{EO}, r^{2}=0.9977\right)$. The decreases in volatile ingredients over time were best described by the following equations: for AT, $Y=0.6557 \mathrm{e}^{-0.316 t}$, for blend of AT and EO, $Y=0.715 \mathrm{e}^{-0.101 \mathrm{t}}$, and for EO, $Y=0.8642 \mathrm{e}^{-0.049 \mathrm{t}}$. Half-life time of dispensers can be calculated by the equation: $t_{1 / 2}=0.693 / k$. Thus, micro-centrifuge tube dispensers with 1 -mL loadings will release $50 \%$ of AT in $\sim 2$ days, EO in $\sim 14$ days, and AT and EO blend (ratio $=1: 1)$ in $\sim 7$ days.

\section{Discussion}

Chemical analyses of the headspace volatiles of apple juices using GC-MS revealed 20 identified aromas, of which some were increased in fermented apple juice and others were only produced by the fermentation. EO was a close-range attractant; it revealed the most powerful attracting capacity to $D$. suzukii in laboratory bioassays, but showed no activity by itself under field conditions. However, addition of EO into the binary blend of AT and AA to form a ternary blend $(\mathrm{AT}+\mathrm{EO}+\mathrm{AA})$ significantly enhanced SWD attraction in the field. Ethanol, which has been previously reported as a principal component for SWD attraction from apple cider vinegar, wine, and yeast baits, did not enhance the activity of AT and AA. However, it did significantly decrease the SWD specificity of the blend (from $\sim 90$ to $\sim 30 \%$ ) by attracting many other nontarget insect species into the traps.

The compound, 3-hydroxy-2-butanone, also known as acetoin, is one of the volatile components found in increased
Fig. 6 Mean $( \pm$ SE) residual amounts of acetoin (AT), ethyl octanoate (EO), and blend of $\mathrm{AT}$ and $\mathrm{EO}$ (ratio=1:1) measured in micro-centrifuge tube dispensers after exposure under fume hood conditions for a period of 2 weeks

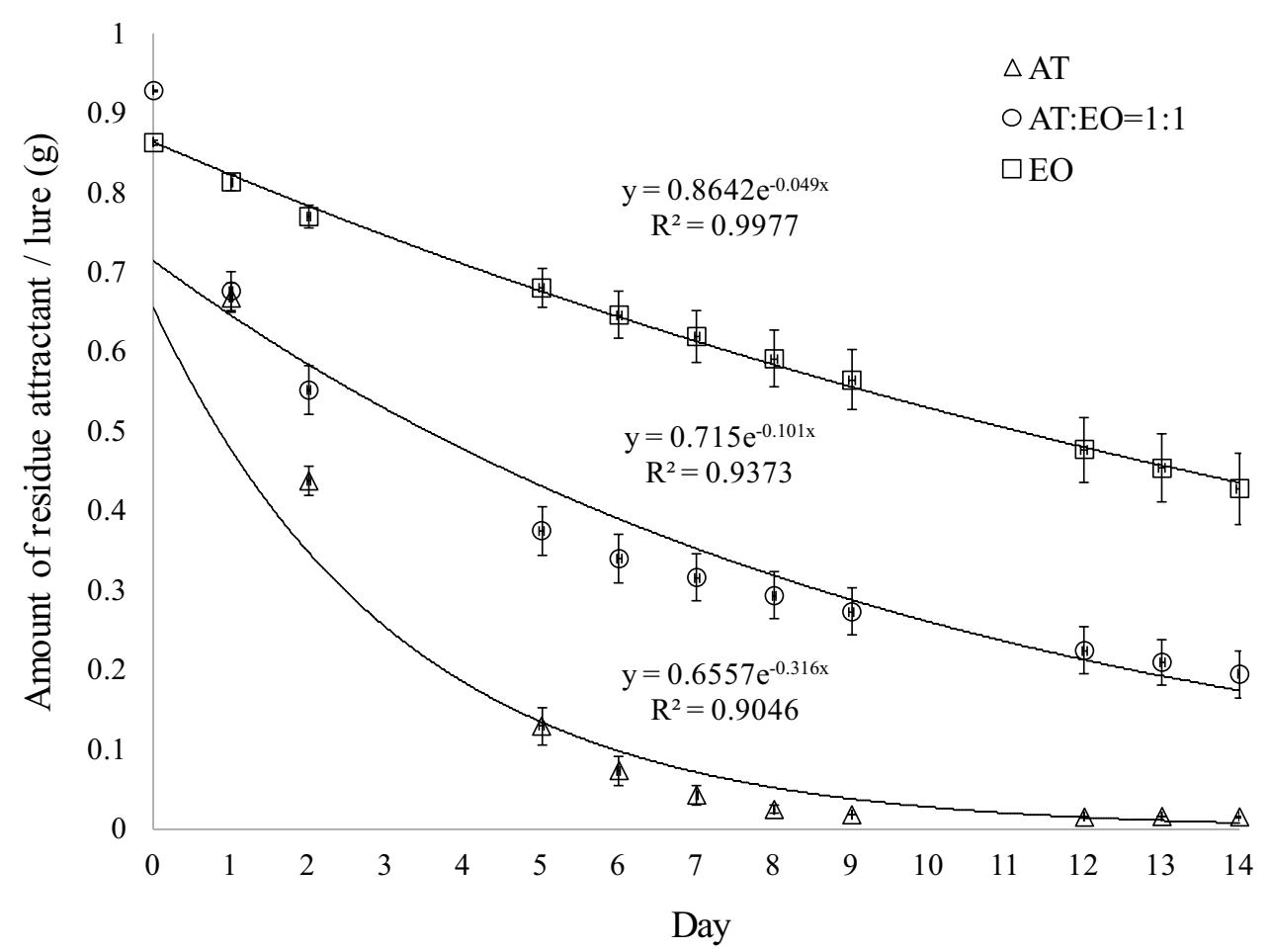


amounts in fermented apple juice. It exists widely in nature and mainly used in baked foods as additive to enhance flavor (Bratovanova 2001; Ensminger et al. 1994; Xiao and Lu 2014a, b). It can be found in apples, butter, yogurt, asparagus, blackcurrants, blackberry, wheat, broccoli, brussels sprouts, and cantaloupe as food flavoring and fragrance (Aili et al. 2012; Bratovanova 1997; de Figueroa et al. 2001) and is an important physiological metabolite excreted by many microorganisms (Romano et al. 1993; Xiao and Xu 2007). Its threshold value in wine is very high, being about $150 \mathrm{mg} / \mathrm{L}$ (Romano and Suzzi 1996). AT has been identified from apple cider as a volatile component (Kahn et al. 1966; Rinaldi et al. 1974). A large amount of AT was produced by the vinegar fermentation produced, while only trace was released by the yeast fermentation of cider (Kahn et al. 1966; Rinaldi et al. 1974). It is similar to our result; a significant amount of AT was produced by the fermented apple juice, while only trace was released by the fresh apple juice (compound 10, Fig. 1). In addition, AT has also been shown as a semiochemical to attract insects (Said et al. 2005; Sreng 1990, 1993; Tolasch et al. 2003; Vlasakova et al. 2008; Witzgall et al. 2010). For SWD, it has been reported that a four-component synthetic bait [acetic acid, ethanol, acetoin, and methionol], in which the EtOH and AA were principal components for activity, was essential for SWD attraction (Cha et al. 2012, 2014, 2015). However, our data revealed that the single compound, AT, showed moderate activity in laboratory bioassays, but was a major semiochemical for SWD long-range attraction in the field.

The compound, ethyl octanoate, is also known as ethyl caprylate. It has been found in wine and produced during the fermentation process by yeast (Antonelli et al. 1999; Gallardo-Chacon et al. 2010; Patel and Shibamoto 2002; Tsakiris et al. 2010; Vianna and Ebeler 2001) and widely used in fragrances, flavorings, pharmaceuticals, and cosmetics. In addition, EO is known as one ingredient in multiple-component attractants for several insects including the fruit flies in the Tephritidae (El-Sayed 2017; Robacker et al. 1992; Toledo et al. 2009). However, to the best our knowledge, ethyl octanoate has not been reported as an attractant component for D. suzukii. Interestingly, although it exhibited the strongest activity for SWD attraction in the laboratory bioassay, it was not attractive to $D$. suzukii in field conditions, indicating that EO is a close-range attractant. It could significantly synergize the attraction of acetoin (AT) in the field.

Attractive activity of the ternary blend (AT + EO + AA) to $D$. suzukii was also significantly increased by adding ethyl acetate to form a quaternary blend $(\mathrm{AT}+\mathrm{EO}+\mathrm{AA}+\mathrm{EA})$. It was 2-4 times more attractive and 2-3 times more selective than the widely used ACV and commercially available SWD lures under field conditions, indicating that the EA is also a significant synergist for SWD attraction.
Moreover, phenethyl alcohol, a volatile enriched during fermentation, also functioned as a strong synergistic agent. Our data indicated that a quinary chemical blend $(\mathrm{AT}+\mathrm{EO}+\mathrm{AA}+\mathrm{EA}+\mathrm{PE})$ was more attractive than the quaternary blend $(\mathrm{AT}+\mathrm{EO}+\mathrm{AA}+\mathrm{EA})$ in field conditions and that neither EA nor PE was attractive to D. suzukii in the field.

Ethyl acetate is a major volatile component in plants (Malkina et al. 2011; Nonato et al. 2001) including many fruits (Krokida and Philippopoulos 2006; Nojima et al. 2003), as well as in wine (Ciani et al. 2006; Plata et al. 2003; Romano et al. 2003; Viana et al. 2008), beer (Jelen et al. 1998), whiskey (Carter and Linsky 1974), microbes (Lobs et al. 2016), and animal waste (Yasuhara 1987). It is an ester of ethanol and acetic acid and has been widely used as flavor enhancer in food and beverage production and as aroma enhancer in cosmetics and perfumes. It is affirmed by the United States Food and Drug Administration as GRAS (generally recognized as safe) (Opdyke 2013) and widely accepted as a safe food additive in many countries with $\mathrm{E}$ number E1504. In addition, EA is also widely used as a solvent for extracting organic compounds. Moreover, EA has also been reported as a component of pheromones or attractants for a variety of insects (El-Sayed 2017). For $D$. suzukii, our data clearly demonstrate that EA can significantly synergize the attraction of the ternary blend in the field; this is in contrast to previous findings that EA acts as a repellent that reduces the attraction of $D$. suzukii to blends of ethanol and acetic acid in both laboratory and field conditions (Cha et al. 2012).

The compound, phenethyl alcohol, occurs widely in nature. It is the main volatile component of rose aromas (Kim et al. 2010) and can also be found in many other essential oils, e.g., carnation, hyacinth, Aleppo pine, orange blossom, ylang-ylang, geranium, neroli, and champaca (Li et al. 2014). Because of its mild rose odor, $P E$ has been extensively used in cosmetics, flavors, and perfumes (Scognamiglio et al. 2012). Interestingly, it also is an autoantibiotic produced by the fungus Candida albicans (Robin) Berkhout (Lingappa et al. 1969). As a common semiochemical, PE has been used by more than 90 different insect/arthropod species in their chemical communication (El-Sayed 2017), notably as a strong repellent against ticks (Thorsell et al. 2006). For D. suzukii, PE is one of the attractive components in the baits used by growers in the Pacific Coast states (Walsh et al. 2011). To the best our knowledge, PE has not been reported as single component to have any synergistic interactions with other components in $D$. suzukii attraction.

Most of the SWD baits published prior to our experiments were based on two major fermentation products, ethanol and acetic acid, and activity of these principal compounds could be synergized by several other chemicals (Cha et al. 2012, 2014, 2015; Kleiber et al. 2014; 
Landolt et al. 2012a, b). However, neither of these two compounds alone or in combination showed significant activities in our studies, although olfactory attraction of $D$. suzukii by symbiotic acetic acid bacteria has been observed in a two-way olfactometer bioassay (Mazzetto et al. 2016). Instead, acetic acid exhibited significantly synergistic effect to the SWD attraction of AT and ethanol had the undesirable effect of reducing lure specificity. In addition, the most common yeast bait and commercial lures had poor selectivity for D. suzukii. By comparing with yeast bait and several commercial available lures in cherry orchards, Kirkpatrick et al. (2017) found that the percentage of total captures in traps ranged from 31 to $39 \%$ for D. suzukii and 60 to $68 \%$ for nontarget flies. However, our quinary chemical blend $(\mathrm{AT}+\mathrm{EO}+\mathrm{AA}+\mathrm{EA}+\mathrm{PE})$ showed much higher selectivity, which could trap $72 \%$ (ChemTica) and $47 \%$ (polypropylene micro-centrifuge tube formulation) $D$. suzukii in the field. This result also indicated that the controlled release rate dispenser had significantly higher activity and selectivity for $D$. suzukii.

Our laboratory release rate data indicated that EO not only functioned as a close-range attractant, but also could slow down the release rate of AT when they were blended together $\left(k_{\mathrm{AT}}=0.316<k_{\mathrm{AT}+\mathrm{EO}}=0.101\right)$, thereby prolonging lure longevity. Considering that the average temperature in the field may be higher than in our laboratory, the actual rate constant $(k)$ could be greater than that obtained in the laboratory. Therefore, the effective half-life for our baits may be shorter in the field than the calculated value. Our laboratory prepared dispensers baited with $1 \mathrm{~mL}$ AT and $\mathrm{EO}$ blend (ratio $=1: 1$ ) as the major SWD attractant in a quaternary blend $(\mathrm{AT}+\mathrm{EO}+\mathrm{AA}+\mathrm{EA})$ or a quinary blend $(\mathrm{AT}+\mathrm{EO}+\mathrm{AA}+\mathrm{EA}+\mathrm{PE})$ could not be expected to provide more than 1 week of maximum SWD attraction under field conditions. Therefore, controlled release rate dispenser will resolve this problem.

Our identified SWD attractant blend based on fermentation may have significant impact on the management of this invasive species. As a demonstrated essential volatile component for SWD attraction, acetoin could be one of the major chemical cues used to attract D. suzukii to bait or monitoring stations. The newly identified quinary chemical blend (AT + EO + EA + AA, PE) will be examined for attract SWD at lower population levels and before extensive fruit injury can occur. In addition, elimination of $\mathrm{EtOH}$ as a bait component will increase lure specificity and allow traps to reduce nontarget captures of pollinators and other beneficial insects. Additional field tests will be conducted in orchards/fields during the growing season with the competitive presence of host fruits. The increased attractiveness and specificity of the quinary chemical blend may help accurately detect SWD adult infestations for timely pest management interventions, thereby reducing the need for conventional insecticide usage and ultimately protecting our environment and ecosystem.

\section{Authors' contributions}

All authors performed experiments. AZ and YF conceived the idea. AZ, YF, RB, and AR wrote the manuscript. All authors contributed to the discussion and reviewed the manuscript.

Acknowledgments We thank Dr. Cesar Rodriguez-Saona, Department of Entomology, Rutgers University, for providing the SWD colony and express gratitude to the Systematic Entomology Laboratory, USDA, ARS, Beltsville, MD, for specimen identification of field-collected flies. We are deeply grateful for the technical assistance of Filadelfo Guzman, Invasive Insect Behavior and Biocontrol Laboratory, USDA, ARS, on GC/GC-MS instrumentation and semiochemical collection. We also extend appreciation to Mr. Ben Butler, assistant farm manager of Butler's Orchard, for the use of their blueberry field for trap studies.

Funding This study was fully funded by USDA. The funder had no role in the study design, data collection and analysis, decision to publish, or preparation of the manuscript.

\section{Compliance with ethical standards}

Conflict of interest The authors declare that they have no conflict of interest. Mention of trade names or commercial products in this article is solely for the purpose of providing specific information and does not imply recommendation or endorsement by the USDA.

Ethical standard Specific permission was not required for collection of SWD in USDA, ARS, Beltsville Agricultural Research Center, Beltsville, MD. The land was owned by our employer, USDA. Permission was obtained from Mr. Ben Butler, assistant farm manager of Butler's Orchard, Germantown, MD, to collect SWD in a blueberry field. The collections made during this study did not involve endangered or protected species.

Data availability The data that support the findings of this study are available from the corresponding author upon reasonable request.

Research involving human participants and/or animals This article does not contain any studies with human participants or animals (vertebrates) performed by any of the authors.

Open Access This article is distributed under the terms of the Creative Commons Attribution 4.0 International License (http://creativeco mmons.org/licenses/by/4.0/), which permits unrestricted use, distribution, and reproduction in any medium, provided you give appropriate credit to the original author(s) and the source, provide a link to the Creative Commons license, and indicate if changes were made. 


\section{References}

Abraham J, Zhang A, Angeli S, Abubeker S, Michel C, Feng Y, Rodriguez-Saona C (2015) Behavioral and antennal responses of spotted wing drosophila, Drosophila suzukii, to volatiles from fruit extracts. Environ Entomol 44:356-367

Aili W, Zaigui L, Huanlu S, Changzhong R (2012) Key aroma compounds in Shanxi aged tartary buckwheat vinegar and changes during its thermal processing. Flavour Fragr J 27:47-53

Antonelli A, Castellari L, Zambonelli C, Carnacini A (1999) Yeast influence on volatile composition of wines. J Agric Food Chem 47:1139-1144

Asplen MK, Anfora G, Biondi A, Daane KM, Gutierrez AP, ZappalÃ L, Choi DS, Chu D, Gibert P, Plantamp C, Ponti L, Hoelmer KA, Hutchison WD, Philips CR, Isaacs R, Jiang ZL, Kápáti Z, Kimura MT, Pascual M, Vétek G, Vogt G, Walton VM, Yu Y, Desneux N (2015) Invasion biology of spotted wing Drosophila (Drosophila suzukii): a global perspective and future priorities. J Pest Sci 88:469-494

Basoalto E, Hilton R, Knight A (2013) Factors affecting the efficacy of a vinegar trap for Drosophila suzikii (Diptera; Drosophilidae). J Appl Entomol 137:561-570

Becher PG, Bengtsson M, Hansson BS, Witzgall P (2010) Flying the fly: long-range flight behavior of Drosophila melanogaster to attractive odors. J Chem Ecol 36:599-607

Becher PG, Flick G, Rozpędowska E, Schmidt A, Hagman A, Lebreton S, Larsson MC, Hansson BS, Piškur J, Witzgall P, Bengtsson M, Thompson K (2012) Yeast, not fruit volatiles mediate Drosophila melanogaster attraction, oviposition and development. Funct Ecol 26:822-828

Beers EH, Van Steenwyk RA, Shearer PW, Coates WW, Grant JA (2011) Developing Drosophila suzukii management programs for sweet cherry in the western United States. Pest Manag Sci 67:1386-1395

Bratovanova P (1997) Diacetyl, acetoin and acetaldehyde in the flavour of doughy part-manufactured goods and bread, prepared with leavens of Lactococci and Lactobacilli. Biotechnol Biotechnol Equip 11:53-59

Bratovanova P (2001) To the issue of formation of acetoin and diacetyl in dough part manufactured products. Biotechnol Biotechnol Equip 15:124-127

Burrack HJ, Asplen M, Bahder L, Collins J, Drummond FA, Guedot C, Isaacs R, Johnson D, Blanton A, Lee JC, Loeb G, RodriguezSaona C, van Timmeren S, Walsh D, McPhie DR (2015) Multistate Comparison of attractants for monitoring Drosophila suzukii (Diptera: Drosophilidae) in blueberries and caneberries. Environ Entomol 44:704-712

Calabria G, Maca J, Bachli G, Serra L, Pascual M (2010) First records of the potential pest species Drosophila suzukii (Diptera: Drosophilidae) in Europe. J Appl Entomol 136:139-147

Carter RV, Linsky B (1974) Gaseous emissions from whiskey fermentation units. Atmos Environ 8:526

Cha D, Adams T, Rogg H, Landolt P (2012) Identification and field evaluation of fermentation volatiles from wine and vinegar that mediate attraction of spotted wing drosophila, Drosophila suzukii. J Chem Ecol 38:1419-1431

Cha DH, Hesler SP, Cowles RS, Vogt H, Loeb GM, Landolt PJ (2013) Comparison of a synthetic chemical lure and standard fermented baits for trapping Drosophila suzukii (Diptera: Drosophilidae). Environ Entomol 42:1052-1060

Cha DH, Landolt PJ, Adams T, Rogg H, Werle CT, Sampson BJ, Adamczyk JJ (2014) A four-component synthetic attractant for Drosophila suzukii (Diptera: Drosophilidae) isolated from fermented bait headspace. Pest Manag Sci 70:324-331
Cha DH, Hesler SP, Park S, Adams TB, Zack RS, Rogg H, Loeb GM, Landolt PJ (2015) Simpler is better: fewer non-target insects trapped with a four-component chemical lure vs. a chemically more complex food-type bait for Drosophila suzukii. Entomol Exp Appl 154:251-260

Ciani M, Beco L, Comitini F (2006) Fermentation behaviour and metabolic interactions of multistarter wine yeast fermentations. Int J Food Microbiol 108:239-245

Cini A, Ioriatti C, Anfora G (2012) A review of the invasion of Drosophila suzukii in Europe and a draft research agenda for integrated pest management. Bull Insectol 65:149-160

Dalton DT, Walton VM, Shearer PW, Walsh DB, Caprile J, Isaacs R (2011) Laboratory survival of Drosophila suzukii under simulated winter conditions of the Pacific Northwest and seasonal field trapping in five primary regions of small and stone fruit production in the United States. Pest Manag Sci 67:1368-1374

de Figueroa RM, Oliver G, de Cardenas ILB (2001) Influence of temperature on flavour compound production from citrate by $\mathrm{Lac}$ tobacillus rhamnosus ATCC 7469. Microbiol Res 155:257-262

El-Sayed AM (2017) The pherobase: database of insect pheromones and semiochemicals [WWW document]. URL http://www.phero base.com

Ensminger AH, Ensminger ME, Konlande JE, Robson JR (1994) Foods and nutrition encyclopedia, vol 1 and 2, 2nd edn. CRC Press Inc, Boca Raton

Gallardo-Chacon JJ, Vichi S, Lopez-Tamames E, Buxaderas S (2010) Changes in the sorption of diverse volatiles by Saccharomyces cerevisiae lees during sparkling wine aging. J Agric Food Chem 58:12426-12430

George D, Mallery P (2002) SPSS for Windows step by step: a simple guide and reference, 4th edn. Allyn \& Bacon, Boston

Goodhue RE, Farnsworth D, Williams JC, Bolda M, Zalom FG (2011) Spotted wing drosophila infestation of California strawberries and raspberries: economic analysis of potential revenue losses and control costs. Pest Manag Sci 67:1396-1402

Hamby KA, Becher PG (2016) Current knowledge of interactions between Drosophila suzukii and microbes, and their potential utility for pest management. J Pest Sci 89:621-630

Hamby KA, Bellamy DE, Chiu JC, Lee JC, Walton VM, York RM, Wiman NG, Biondi A (2016) Biotic and abiotic factors impacting development, behavior, phenology, and reproductive biology of Drosophila suzukii. J Pest Sci 89:605-619

Hauser M (2011) A historic account of the invasion of Drosophila suzukii (Matsumura) (Diptera: Drosophilidae) in the continental United States, with remarks on their identification. Pest Manag Sci 67:1352-1357

Iglesias LE, Nyoike TW, Liburd OE (2014) Effect of trap design, bait type, and age on captures of Drosophila suzukii (Diptera: Drosophilidae) in berry crops. J Econ Entomol 107:1508-1518

Jelen HH, Wlazly K, Wasowicz E, Kaminski E (1998) Solid-phase microextraction for the analysis of some alcohols and esters in beer: comparison with static headspace method. J Agric Food Chem 46:1469-1473

Kahn JH, Nickol GB, Conner HA (1966) Vinegar compounds: analysis of vinegar by gas-liquid chromatography. J Agric Food Chem $14: 460-465$

Kim JH, Choi DK, Lee SS, Choi SJ, Kim CD, Yoon TJ, Lee JH (2010) Enhancement of keratinocyte differentiation by rose absolute oil. Ann Dermatol 22:255-261

Kirkpatrick DM, McGhee PS, Gut LJ, Miller JR (2017) Improving monitoring tools for spotted wing drosophila, Drosophila suzukii. Entomol Exp Appl 164:87-93

Kleiber JR, Unelius CR, Suckling DM, Lee JC, Qian MC, Bruck DJ (2014) Attractiveness of fermentation and related products to spotted wing drosophila (Diptera: Drosophilidae). Environ Entomol 43:439-447 
Krokida MK, Philippopoulos C (2006) Volatility of apples during air and freeze drying. J Food Eng 73:135-141

Landolt PJ, Adams T, Rogg H (2012a) Trapping spotted wing drosophila, Drosophila suzukii (Matsumura) (Diptera: Drosophilidae), with combinations of vinegar and wine, and acetic acid and ethanol. J Appl Entomol 136:148-154

Landolt PJ, Davis TS, Adams T, Rogg H (2012b) Spotted wing drosophila, Drosophila suzukii (Diptera: Drosophilidae), trapped with combinations of wines and vinegars. Florida Entomol 95:326-332

Lee JC, Bruck DJ, Dreves AJ, Ioriatti C, Vogt H, Baufeld P (2011) In focus: spotted wing drosophila, Drosophila suzukii, across perspectives. Pest Manag Sci 67:1349-1351

Lee JC, Bruck DJ, Burrack HJ, Walsh DB, Barrantes LD, Beers EH, Dreves AJ, Hamby KA, Zalom FG, Haviland DR, Isaacs R, Richardson TA, Shearer PW, Stanley CA, Walton VM (2012) Evaluation of monitoring traps for Drosophila suzukii (diptera: Drosophilidae) in North America. J Econ Entomol 105:1350-1357

Li C, Sun JC, Li TH, Liu SQ, Huang DJ (2014) Chemical and enzymatic synthesis of a library of 2-phenethyl esters and their sensory attributes. Food Chem 154:205-210

Lingappa BT, Prasad M, Lingappa Y, Hunt DF, Biemann K (1969) Phenethyl alcohol and tryptophol: autoantibiotics produced by the fungus Candida albicans. Science 163:192-194

Lobs AK, Lin JL, Cook M, Wheeldon I (2016) High throughput, colorimetric screening of microbial ester biosynthesis reveals high ethyl acetate production from Kluyveromyces marxianus on C5, C6, and C12 carbon sources. Biotechnol J 11:1274-1281

Malkina IL, Kumar A, Green PG, Mitloehner FM (2011) Identification and quantitation of volatile organic compounds emitted from dairy silages and other feedstuffs. J Environ Qual 40:28-36

Mallis A (1969) Handbook of pest control, 5th edn. Mac NairDorland Co., New York

Mazzetto F, Pansa MG, Ingegno BL, Tavella L, Alma A (2015) Monitoring of the exotic fly Drosophila suzukii in stone, pome and soft fruit orchards in NW Italy. J Asia Pac Entomol 18:321-329

Mazzetto F, Gonella E, Pontini M, Alma A, Crotti E, Vacchini V, Daffonchio D, Syrpas M, Mangelinckx S (2016) Olfactory attraction of Drosophila suzukii by symbiotic acetic acid bacteria. J Pest Sci 89:783-792

Mitsui H, Takahashi KH, Kimura MT (2006) Spatial distributions and clutch sizes of Drosophila species ovipositing on cherry fruits of different stages. Popul Ecol 48:233-237

Nojima S, Linn C, Roelofs W (2003) Identification of host fruit volatiles from flowering dogwood (Cornus florida) attractive to dogwoodorigin Rhagoletis pomonella flies. J Chem Ecol 29:2347-2357

Nonato EA, Carazza F, Silva FC, Carvalho CR, Cardeal ZD (2001) A headspace solid-phase microextraction method for the determination of some secondary compounds of Brazilian sugar cane spirits by gas chromatography. J Agric Food Chem 49:3533-3539

Opdyke DLJ (2013) Monographs on fragrance raw materials. A collection of monographs originally appearing in food and cosmetics toxicology. In: Opdyke DLJ (ed) Monographs on fragrance raw materials. Pergamon Press, Oxford, p 804

Patel S, Shibamoto T (2002) Effect of different strains of Saccharomyces cerevisiae on production of volatiles in Napa Gamay wine and Petite Sirah wine. J Agric Food Chem 50:5649-5653

Plata C, Millan C, Mauricio JC, Ortega JM (2003) Formation of ethyl acetate and isoamyl acetate by various species of wine yeasts. Food Microbiol 20:217-224

Rinaldi G, Meinschein WG, Hayes JM (1974) Intramolecular carbon isotopic distribution in biologically produced acetoin. Biomed Mass Spectrom 1:415-417

Robacker DC, Warfield WC, Flath RA (1992) A four-component attractant for the Mexican fruit fly, Anastrepha ludens (Diptera: Tephritidae), from host fruit. J Chem Ecol 18:1239-1254
Romano P, Suzzi G (1996) Origin and production of acetoin during wine yeast fermentation. Appl Environ Microbiol 62:309-315

Romano P, Suzzi G, Zironi R, Comi G (1993) Biometric study of acetoin production in Hanseniaspora guilliermondii and Kloeckera apiculata. Appl Environ Microbiol 59:1838-1841

Romano P, Fiore C, Paraggio M, Caruso M, Capece A (2003) Function of yeast species and strains in wine flavour. Int J Food Microbiol 86:169-180

Said I, Renou M, Morin JP, Ferreira JMS, Rochat D (2005) Interactions between acetoin, a plant volatile, and pheromone in Rhynchophorus palmarum: behavioral and olfactory neuron responses. J Chem Ecol 31:1789-1805

Scognamiglio J, Jones L, Letizia CS, Api AM (2012) Fragrance material review on phenylethyl alcohol. Food Chem Toxicol 50:S224-S239

Sokal RR, Rohlf FJ (1995) Biometry: the principles and practice of statistics in biological research, 3rd edn. W.H. Freeman and Company, New York

Sreng L (1990) Seducin, male sex pheromone of the cockroach Nauphoeta cinerea: isolation, identification, and bioassay. J Chem Ecol 16:2899-2912

Sreng L (1993) Cockroach mating behaviors, sex pheromones, and abdominal glands (Dictyoptera: Blaberidae). J Insect Behav 6:715-735

Thorsell W, Mikiver A, Tunon H (2006) Repelling properties of some plant materials on the tick Ixodes ricinus L. Phytomedicine 13:132-134

Tolasch T, Solter SR, Toth M, Ruther J, Francke W (2003) (R)-acetoinfemale sex pheromone of the summer chafer Amphimallon solstitiale (L). J Chem Ecol 29:1045-1050

Toledo J, Malo EA, Cruz-Lpez L, Rojas JC (2009) Field evaluation of potential fruit-derived lures for Anastrepha obliqua (Diptera: Tephritidae). J Econ Entomol 102:2072-2077

Tonina L, Grassi A, Caruso S, Mori N, Gottardello A, Anfora G, Giomi F, Vaccari G, Ioriatti C (2018) Comparison of attractants for Drosophila suzukii monitoring in sweet cherry orchards in Italy. J Appl Entomol 142:18-25

Tsakiris A, Koutinas AA, Psarianos C, Bekatorou A, Kourkoutas Y (2010) A new process for wine production by penetration of yeast in uncrushed frozen grapes. Appl Biochem Biotechnol 162:1109-1121

Viana F, Gil JV, Genoves S, Valles S, Manzanares P (2008) Rational selection of non-Saccharomyces wine yeasts for mixed starters based on ester formation and enological traits. Food Microbiol 25:778-785

Vianna E, Ebeler SE (2001) Monitoring ester formation in grape juice fermentations using solid phase microextraction coupled with gas chromatography-Mass spectrometry. J Agric Food Chem 49:589-595

Vlasakova B, Kalinova B, Gustafsson MHG, Teichert H (2008) Cockroaches as pollinators of Clusia aff. sellowiana (Clusiaceae) on inselbergs in French Guiana. Ann Bot 102:295-304

Walsh DB, Bolda MP, Goodhue RE, Dreves AJ, Lee J, Bruck DJ, Walton VM, O'Neal SD, Zalom FG (2011) Drosophila suzukii (Diptera: Drosophilidae): Invasive pest of ripening soft fruit expanding its geographic range and damage potential. J Integr Pest Manag 2:G1-G7

Wang XG, Stewart TJ, Biondi A, Chavez BA, Daane KM, Ingels C, Caprile J, Grant JA, Walton VM (2016) Population dynamics and ecology of Drosophila suzukii in Central California. J Pest Sci 89:701-712

Witzgall P, Kirsch P, Cork A (2010) Sex pheromones and their impact on pest management. J Chem Ecol 36:80-100

Xiao Z, Lu JR (2014a) Strategies for enhancing fermentative production of acetoin: a review. Biotechnol Adv 32:492-503

Xiao ZJ, Lu JR (2014b) Generation of Acetoin and its derivatives in foods. J Agric Food Chem 62:6487-6497

Xiao ZJ, Xu P (2007) Acetoin metabolism in bacteria. Crit Rev Microbiol 33:127-140 
Yasuhara A (1987) Identification of volatile compounds in poultry manure by gas chromatography-mass spectrometry. J Chromatogr 387:371-378

Zhang A, Facundo HT, Robbins PS, Linn CE Jr, Hanula JL, Villani MG, Roelofs WL (1994) Identification and synthesis of female sex pheromone of Oriental beetle, Anomala orientalis (Coleoptera: Scarabaeidae). J Chem Ecol 20:2415-2427

Zhang A, Linn C Jr, Wright S, Prokopy R, Reissig W, Roelofs W (1999) Identification of a new blend of apple volatiles attractive to the apple maggot, Rhagoletis pomonella. J Chem Ecol 25:1221-1232
Zhang A, Amalin D, Shirali S, Serrano MS, Franqui RA, Oliver JE, Klun JA, Aldrich JR, Meyerdirk DE, Lapointe SL (2004) Sex pheromone of the pink hibiscus mealybug, Maconellicoccus hirsutus, contains an unusual cyclobutanoid monoterpene. Proc Natl Acad Sci USA 101:9601-9606 